\title{
biniou $(F o x F)$, a central component in a regulatory network controlling visceral mesoderm development and midgut morphogenesis in Drosophila
}

\author{
Stephane Zaffran, ${ }^{1}$ Axel Küchler, ${ }^{2}$ Hsiu-Hsiang Lee, and Manfred Frasch ${ }^{3}$ \\ Department of Biochemistry and Molecular Biology, Mount Sinai School of Medicine, New York, New York 10029, USA
}

The subdivision of the lateral mesoderm into a visceral (splanchnic) and a somatic layer is a crucial event during early mesoderm development in both arthropod and vertebrate embryos. In Drosophila, this subdivision leads to the differential development of gut musculature versus body wall musculature. Here we report that biniou, the sole Drosophila representative of the FoxF subfamily of forkhead domain genes, has a key role in the development of the visceral mesoderm and the derived gut musculature. biniou expression is activated in the trunk visceral mesoderm primordia downstream of $d p p$, tinman, and bagpipe and is maintained in all types of developing gut muscles. We show that biniou activity is essential for maintaining the distinction between splanchnic and somatic mesoderm and for differentiation of the splanchnic mesoderm into midgut musculature. biniou is required not only for the activation of differentiation genes that are expressed ubiquitously in the trunk visceral mesoderm but also for the expression of $d p p$ in parasegment 7 , which governs proper midgut morphogenesis. Activation of $d p p$ is mediated by specific Biniou binding sites in a $d p p$ enhancer element, which suggests that Biniou serves as a tissue-specific cofactor of homeotic gene products in visceral mesoderm patterning. Based upon these and other data, we propose that the splanchnic mesoderm layers in Drosophila and vertebrate embryos are homologous structures whose development into gut musculature and other visceral organs is critically dependent on FoxF genes.

[Key Words: Visceral mesoderm; splanchnic mesoderm; midgut; mesoderm induction; forkhead/winged helix domain]

Received June 6, 2001; revised version accepted September 7, 2001.

The musculature of higher organisms consists of three major categories of muscle types: skeletal, cardiac, and visceral muscle. These types of muscle exhibit significant differences with respect to their developmental origins, ultrastructure, contractile properties, and physiological functions. Visceral muscles, which are the focus of the present study, are important components of many different internal organ systems, particularly the gastrointestinal and urogenital tracts, respiratory tract, and vascular system. In Drosophila, the visceral musculature is less diverse and primarily consists of the musculature of the digestive tract. Similar to vertebrates, the musculature of the Drosophila midgut is composed of an inner layer of circular muscles and an outer layer of longitudinal muscles (Campos-Ortega and Hartenstein 1997). Ver-

Present addresses: ${ }^{1}$ Department de Biologie Moleculaire, Institut Pasteur, 75015 Paris, France; ${ }^{2}$ Artemis Pharmaceuticals, 72076 Tübingen, Germany.

${ }^{3}$ Corresponding author.

E-MAIL manfred.frasch@mssm.edu; FAX (212) 860-9279.

Article and publication are at http://www.genesdev.org/cgi/doi/10.1101/ gad.917101. tebrate and Drosophila visceral musculatures have additional similarities, including the features that the fibers are spindle-shaped and display slow, supercontracting properties. Although Drosophila visceral muscles are striated, their striation is atypical, and several of their ultrastructural features are reminiscent of vertebrate smooth muscles (Sandborn et al. 1967; Goldstein and Burdette 1971).

In both vertebrates and arthropods, the visceral mesoderm is largely derived from the lateral (splanchnic) mesoderm, which is under the inductive influence of BMP/ Dpp signals (Holley and Ferguson 1997). Previous studies of Drosophila have shown that Dpp, which is secreted from the dorsal ectoderm, is critically required for the induction of visceral muscle in the underlying lateral (in insects dorsal) mesoderm (Frasch 1995). The mesodermal response to Dpp consists of the transcriptional activation of at least two important regulatory genes in the dorsal mesoderm, which are the NK homeobox genes tinman (tin) and bagpipe (bap) (Staehling-Hampton et al. 1994; Frasch 1995). tin is genetically required for the formation of all dorsal mesodermal derivatives (heart, 
dorsal body wall muscles, and midgut visceral muscles), whereas bap is required exclusively for the specification of the visceral (specifically the circular) muscles of the midgut and functions downstream and as a direct target of tin (Azpiazu and Frasch 1993; Bodmer 1993; Yin and Frasch 1998; H.-H. Lee and M. Frasch, unpubl.). bap is therefore the first example of a regulator that is uniquely involved in the specification of visceral muscles.

The functional differences between tin and bap are reflected in their patterns of expression in the dorsal mesoderm. tin is induced by Dpp in the entire dorsal mesoderm, whereas the induction of bap occurs in segmentally repeated clusters of cells within this area, which represent the primordia of the circular musculature of the midgut (Azpiazu and Frasch 1993). Subsequent cell shape changes lead to the anterior-posterior extension and coalescence of bap-expressing cell clusters into a continuous visceral mesoderm layer, the splanchnopleura, which ultimately surrounds the midgut to form the circular midgut musculature. bap is also expressed in primordial cells of the foregut and hindgut muscles, which form independently of Dpp near the anterior and posterior gut invagination sites, respectively. However, it is not expressed in longitudinal gut muscle precursors, which are specified in the caudal mesoderm, adjacent to the hindgut muscle progenitors. Longitudinal gut muscle formation requires anterior migration and dispersion of these cells along the entire midgut prior to their differentiation (Campos-Ortega and Hartenstein 1997).

Both tin and bap are expressed transiently in the dorsal and visceral mesoderm, and their protein products disappear upon the coalescence of the visceral mesoderm primordia. Because gut muscle morphogenesis and differentiation occur significantly later in development, it would appear that tin and bap are mainly required for visceral mesoderm specification and are not sufficient for the differentiation steps. It is likely that the combination of these two genes, or bap alone, activates the expression of downstream genes that are required to regulate various differentiation events. These events include the expression of adhesion/guidance molecules that direct the invagination of the anterior and posterior endoderm towards the center of the embryo, the subsequent migration and spreading of visceral mesoderm around the gut endoderm, the expression of contractile proteins, and the acquisition of differential properties along the anterior/posterior axis that determine the looping and overall morphogenesis of the midgut. This latter patterning process involves the spatially restricted expression of homeotic genes and signaling molecules, particularly Dpp and Wingless, within the developing visceral mesoderm (Bienz 1994).

In the present study, we describe a new gene, named biniou (bin), which functions as a critical regulator of visceral mesoderm development. biniou encodes a forkhead domain transcription factor of the FoxF subfamily and is expressed throughout embryonic development in all types of gut muscles and their progenitors. We show that the visceral mesoderm fails to differentiate in the absence of biniou activity and is partially transformed into somatic mesoderm. Conversely, ectopic expression of biniou in the somatic mesoderm results in the ectopic activation of visceral mesoderm markers within this layer. We further demonstrate that Biniou binds to a previously described enhancer of the $d p p$ gene and is required in conjunction with the homeotic gene products Ubx and Exd to activate $d p p$ expression in parasegment 7 of the visceral mesoderm, which in turn controls midgut morphogenesis.

\section{Results}

biniou is the single Drosophila representative of the FoxF subfamily of forkhead domain genes

biniou (bin) cDNA encodes a 676 amino acid protein which includes a forkhead (winged helix) domain in its central portion (Fig. 1; see Materials and Methods). Sequence comparisons show that Biniou (Bin) is the only fly representative of the FoxF subfamily of forkhead domain proteins and is equally related to FoxF1 and FoxF2. The sequence similarities between Bin and its vertebrate orthologs are confined to the forkhead domains (Fig. 1A).

The bin gene maps to 65DE on the third chromosome and consists of four exons (Fig. 1B). Among lethal mutations that were obtained in a saturation screen at this location (Anderson et al. 1995), we identified one complementation group with three EMS-induced alleles that correspond to bin. Sequencing of the bin loci from the respective mutant chromosomes confirmed that all three alleles, $\operatorname{bin}^{I 1}, b_{i n}{ }^{S 4}$, and $\operatorname{bin}^{R 22}$, carry mutations in the bin open reading frame (ORF). The mutations map to three different positions within the first exon and in each case introduce a transition from a CAG (Gln) to TAG (Stop) codon (Fig. 1B,C). Because the encoded polypeptides are truncated $\mathrm{N}$-terminally to the forkhead domain and are expected to lack any DNA-binding activity, all three alleles are likely null alleles.

bin is expressed in all types of visceral muscles and their progenitors

Whole-mount stainings of stage 10 embryos with labeled bin cDNA probes and Bin antibodies revealed that bin is expressed in progenitors of all three types of visceral musculature (Fig. 2A,B). These include the 11 bilateral patches of circular midgut muscle progenitors in the dorsal mesoderm in parasegments (PS)2-12 (also known as trunk visceral mesoderm progenitors), the hindgut and foregut visceral mesoderm, as well as the caudal visceral mesoderm which is located just anteriorly to the hindgut visceral mesoderm (Fig. 2A). Double stainings of early embryos with Bin and Bap antibodies showed that the expression of Bin and Bap is activated almost simultaneously in the trunk visceral mesoderm progenitors (Fig. $2 \mathrm{~B}, \mathrm{~B}^{\prime}$ ). However, at early stage 10 , we occasionally detect cells that express Bap but not Bin (Fig. 2B'), indicating that bap expression initiates shortly before bin. We note that bin is expressed in an additional, smaller group 

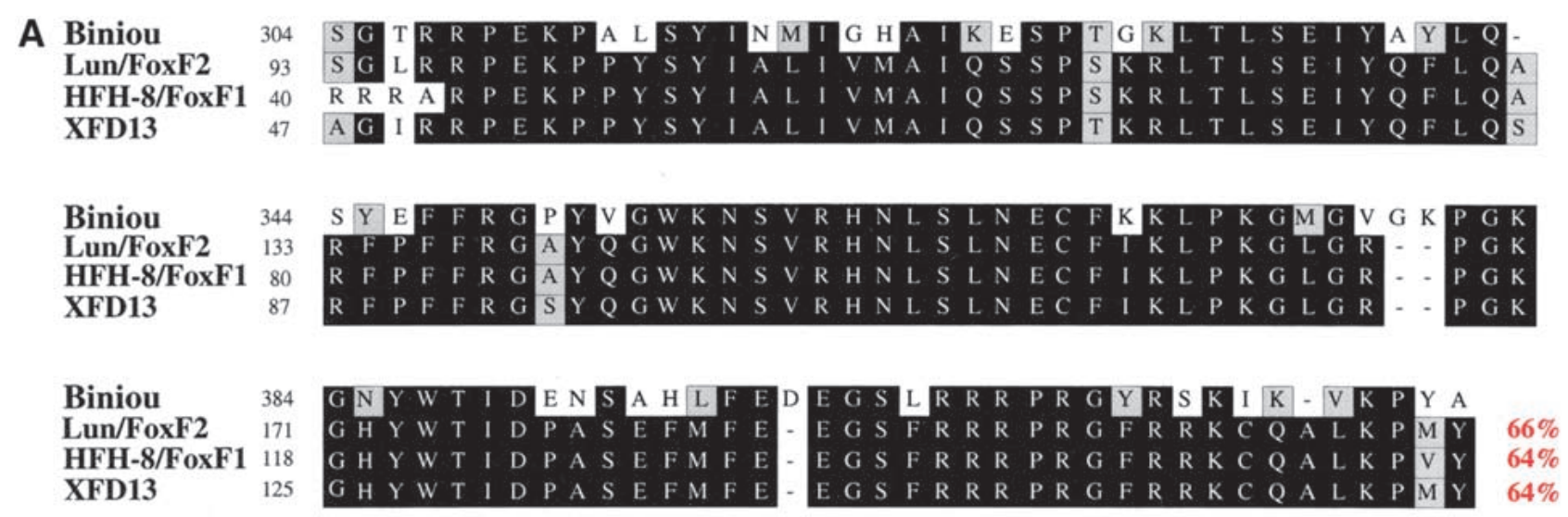

B

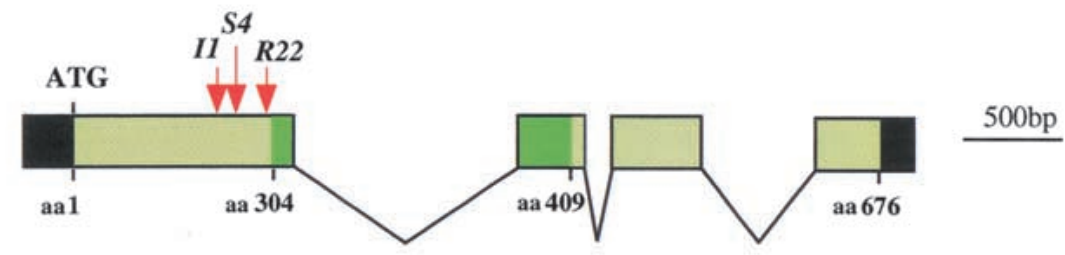

C

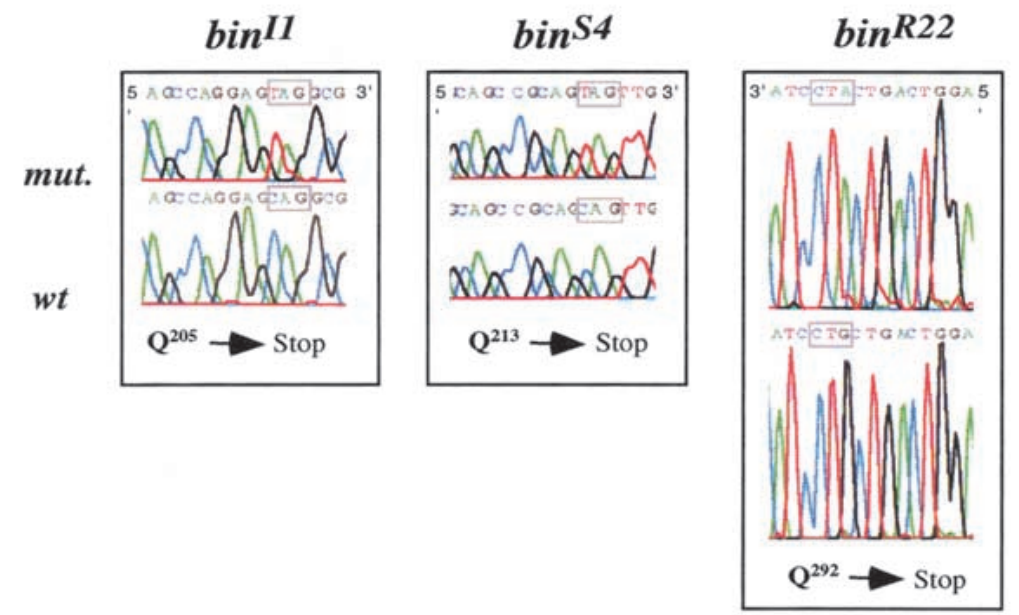

Figure 1. Molecular organization of the biniou locus and characterization of the mutant alleles. (A) Amino acid alignments of the forkhead domain of Biniou (GenBank AAK97051) with those of murine FoxF2 (Lun, amino acids 93-206; GenBank NM_010225), FoxF1 (HFH-8, amino acids 40-155; GenBank I49735), and Xenopus XFD13 (amino acids 47-161; GenBank CAB44732). Identical residues are boxed in black (see percent identity, in red), and conservative changes are shaded lightly. (B) Schematic representation of the genomic structure of the bin locus at 65D with intron-exon structure and ORF. Green and black boxes represent coding and noncoding sequences, respectively (dark green: forkhead domain). The locations of EMS mutations in bin (I1, S4, and R22 alleles) are indicated

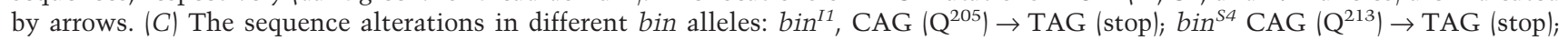
$\operatorname{bin}^{R 22}$ CAG $\left(Q^{292}\right) \rightarrow$ TAG (stop).

of dorsal mesodermal cells in PS 1, which upon coalescence of the metameric cell clusters also becomes part of the trunk visceral mesoderm (Fig. 2A,C). An important difference between bin and bap is that after the coalescence and segregation of the trunk visceral mesoderm, bap expression becomes segmental and then disappears, whereas bin is maintained uniformly and at constant levels. The circular midgut muscle precursors, which at stage 14 form a dorsal row and a ventral row and then surround the midgut, as well as the foregut and hindgut muscles continue to express bin until late embryogenesis (Fig. 2D,E).
The early expression of bin in all visceral mesoderm derivatives allowed us to examine the dynamic morphogenetic changes and compare the gene activities in these developing tissues. Double stainings for Bin and Bap showed that, in contrast to the trunk visceral mesoderm, the hindgut visceral mesoderm expresses bin shortly before bap, and the caudal visceral mesoderm does not express Bap at any time (Fig. 2F-H). The caudal visceral mesoderm splits bilaterally and then each group of cells moves towards and onto the posteriormost patch of trunk visceral mesoderm progenitors (Fig. 2F-H). The nuclei of the caudal visceral mesoderm cells retain Bin 

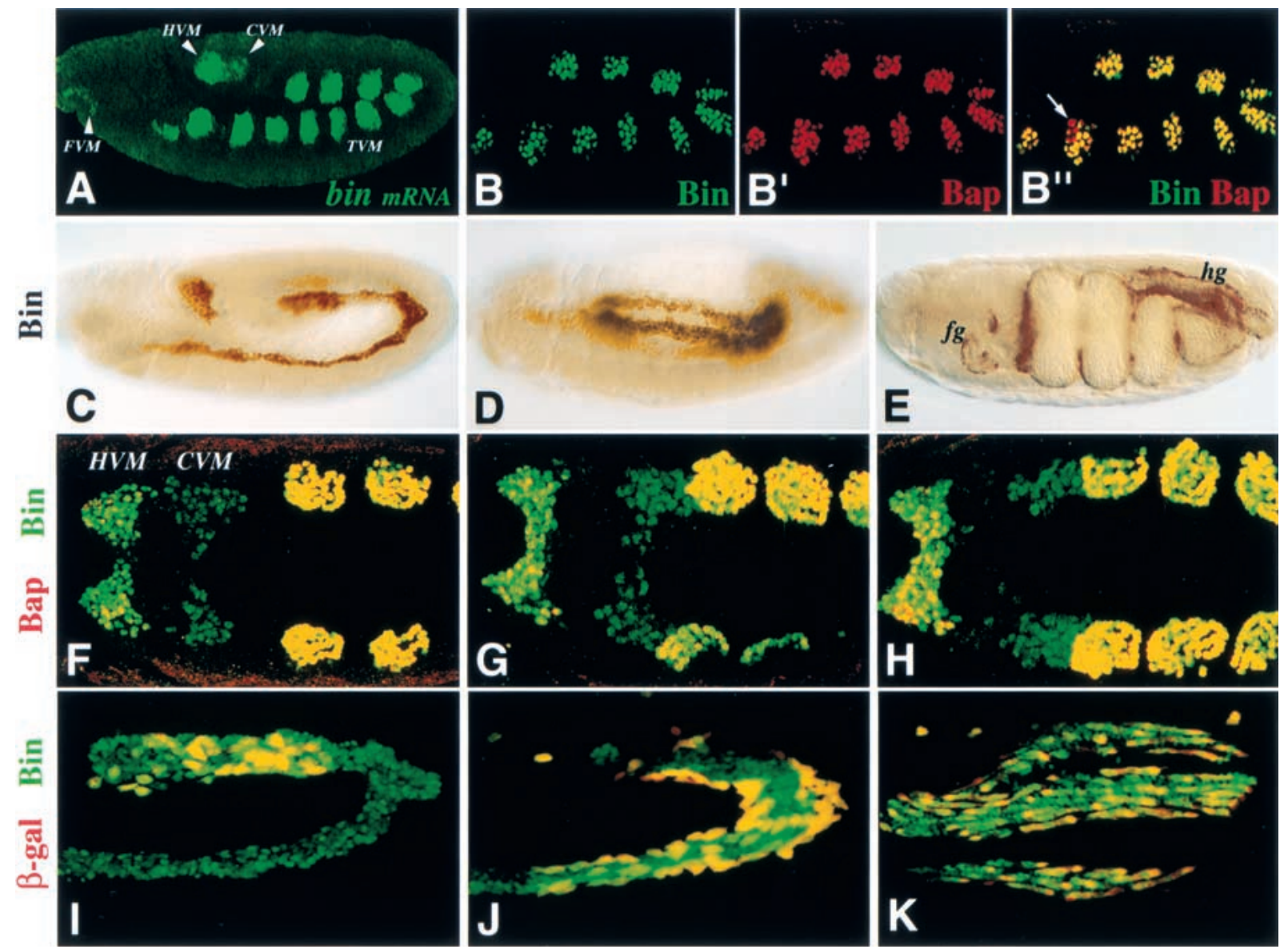

Figure 2. Expression of biniou in wild-type embryos and morphogenesis of caudal visceral mesoderm. Lateral $(A-E, I-K)$ and dorsal $(F-H)$ views of whole-mount embryos are shown. Embryo in $A$ was hybridized with bin mRNA probe using TSA-FITC detection; embryos in $B-K$ were stained with an anti-Bin antibody; embryos in $B^{\prime}$ and $F-H$ were doubly labeled for Bin and Bap, and embryos in $I-K$ for Bin and LM.m4-lacZ-driven $\beta$-gal (marks CVM; H.-H. Lee, unpubl.). (A) bin expression is initiated at stage 10 in 11 patches in the dorsal mesoderm (primordia of the circular midgut muscles), in the foregut and hindgut visceral mesoderm primordia and in the caudal visceral mesoderm (primordium of longitudinal visceral muscles). ( $\left.B-B^{\prime \prime}\right)$ Early stage 10 embryo showing colocalization of Bin protein (green in $B$ ) and Bap protein (red in $\left.B^{\prime}\right)$. Merged image of $B$ and $B^{\prime}\left(B^{\prime \prime}\right)$ shows exclusive expression of Bap protein (red) in some cells (arrow). (C) Early stage 12 embryo with persistent Bin protein expression in the trunk and hindgut visceral mesoderm. (D) Stage 13 embryo; Bin is expressed in the circular as well as longitudinal precursors of midgut muscles. (E) Stage 16 embryo; Bin is expressed in foregut (fg), midgut and hindgut (hg) visceral muscles. $(F-H)$ Colocalization (yellow signal) of Bap (red) and Bin (green) protein in dorsal mesoderm and hindgut mesoderm but not in the caudal visceral mesoderm (CVM). The three different caudal views illustrate the CVM movement relative to the other derivatives during stage 11. (I-K) High-magnification view of embryos at mid-stage 12, late-stage 12, and stage 15. Migrating longitudinal muscle precursors coexpress Bin and $\beta$-gal (yellow), whereas circular muscle precursors expresses only Bin (green). (CVM) caudal; (HVM) hindgut; (TVM) trunk visceral mesoderm.

during their migration and dispersion along the trunk visceral mesoderm until they differentiate into longitudinal midgut muscles (Fig. 2I-K).

\section{bin expression in the trunk visceral mesoderm} primordia is controlled by tin, bap, and dpp

Previous studies have shown that bap-expressing domains are defined by the intersecting dorsal activities of dpp/tin, which act positively, and segmentally modulated activities of $w g / s l p$, which have repressing effects (Azpiazu and Frasch 1993; Staehling-Hampton et al. 1994; Frasch 1995; Azpiazu et al. 1996; Riechmann et al.
1997; Lee and Frasch 2000). As shown in Figure 3B (cf. Fig. 3A), bin also requires tin activity for normal expression in the trunk visceral mesoderm primordia. Whereas bap expression is virtually absent in these cells upon loss of tin activity (Azpiazu and Frasch 1993), residual bin expression is observed in small clusters of cells. To test the possibility that residual expression of bin in tin mutant embryos is due to direct inputs from Dpp, we examined bin expression in embryos in which $d p p$ expression was induced ectopically in the entire mesoderm. Ectopic $d p p$ in a wild-type background, which causes tin expression to be expanded ventrally (Frasch 1995), results in an analogous expansion of the bin domains (Fig. 
Zaffran et al.

Figure 3. Regulation of bin and bap expression in the trunk visceral mesoderm (TVM) primordia. (A) Stage 10 wild-type embryo stained for Bin protein. (B) Stage 10 tin $^{346}$ mutant embryo with strongly reduced Bin expression in the TVM primordia but normal expression in the hindgut visceral mesoderm. $(C)$ Ventrolateral view of stage 10 embryo with ectopic mesodermal $d p p$ expression (driven by twistGAL4). The segmented Bin expression has expanded to the ventral midline (cf. $A)$. $(D)$ Ventrolateral view of stage $10 \operatorname{tin}^{346} \mathrm{mu}-$ tant embryo with ectopic mesodermal $d p p$ expression. Stripes of Bin have expanded to the ventral domain but are much weaker than in a wild-type background (cf. $C)$. (E) Stage 10 bap $^{D f}$ mutant embryo with strongly reduced bin mRNA expression. $(F)$ Ventrolateral view of stage 10 bin mutant embryo in which bin mRNA expression has disappeared in most of the TVM primordia, except for parasegments 1 and 2. $(G, H)$ Stage 11 embryo with uniform bap expression driven by twist-GAL4 in mesoderm. Expansion of Bap expression (red) is accompanied by the expansion of Bin (green) (see $H$ ). (I) Stage 11 wild-type embryo stained for Bap protein (red). Bap is expressed in the forming midgut, hindgut (arrowhead), and foregut (arrow) visceral mesoderm. (J) Stage 11 bin embryo stained for Bap protein. Bap expression (red) is prematurely lost in the midgut mesoderm but not in foregut (arrow) and hindgut (arrowhead) mesoderm (cf. I).
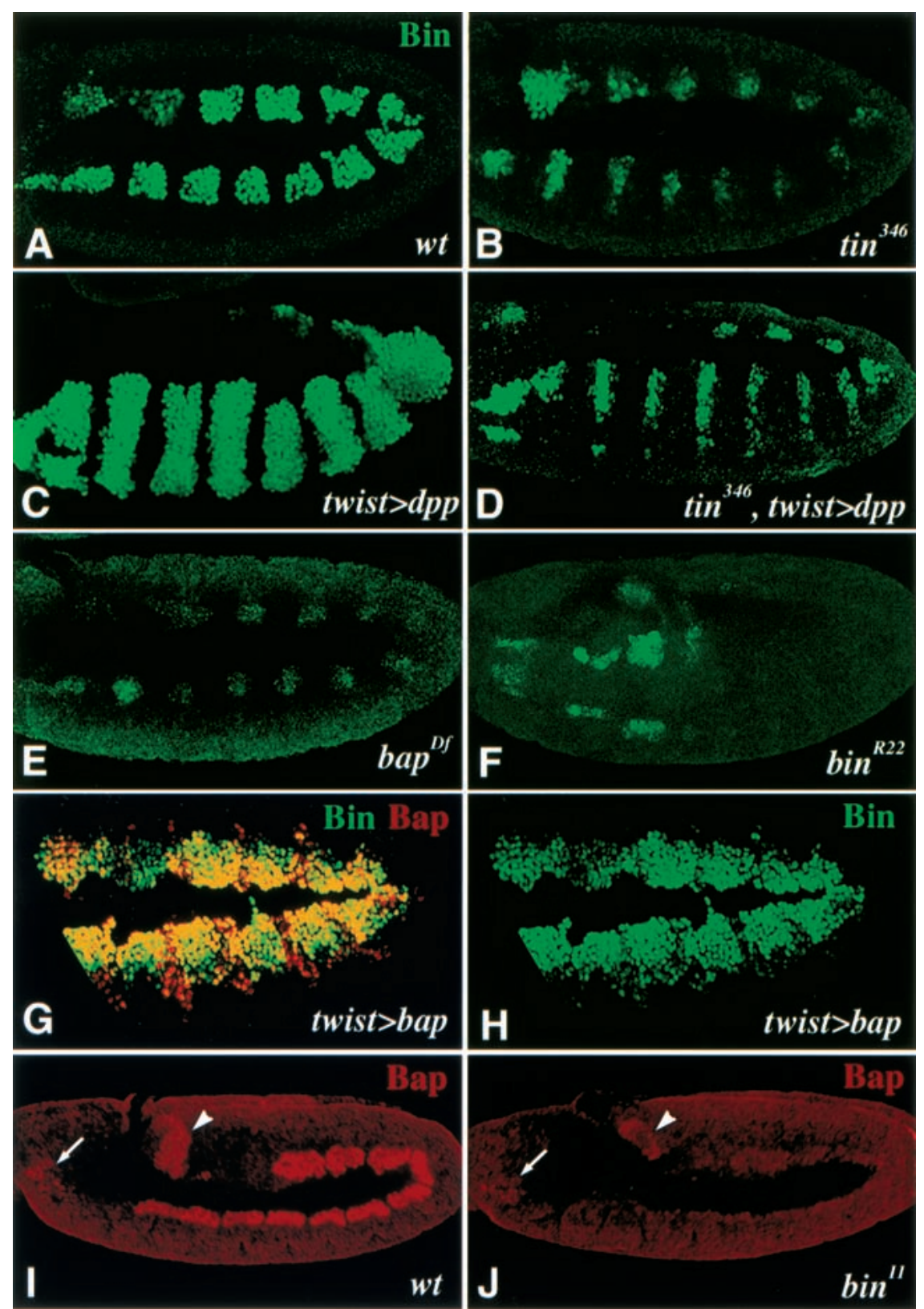

3C). Notably, ventral expansion of the bin domains is also observed upon ectopic $d p p$ expression in the absence of tin activity, although the domains are narrow (Fig. 3D, cf. B and C). Thus, Dpp is able to induce bin in the absence of tin, although tin activity is required for normal expression levels. The residual expression of bin in tin mutant embryos is unstable and not maintained in later stages of development (data not shown).

Similar to tin, bap activity is also required for normal bin expression (Fig. 3E). This result is in agreement with the temporal sequence of bap and bin expression (see above; Fig. 2B') and with the observed expansion of bin throughout most of the dorsal mesoderm upon ectopic bap expression in the mesoderm (Fig. 3G,H). These data suggest that bin is furthest downstream within a mesoderm-intrinsic cascade of gene activation: twist $\rightarrow$ tin $\rightarrow$ bap $\rightarrow$ bin. Moreover, bin itself is required for normal bin expression. Although bin expression initiates normally in stage 10 bin mutant embryos, it disappears at early stage 11 in the trunk visceral mesoderm primordia of bin mutants, except for those in PS1 and 2 (Fig. 3F; data not shown). bin expression in these two parasegments is also less sensitive to the loss of tin and bap activity (Fig. 3B,E). Furthermore, the expression of bin in foregut, hindgut, and caudal visceral mesoderm does not depend on any of the genes examined in the present study.

Whereas the above data show that maintenance of bin expression in most of the presumptive trunk visceral mesoderm requires positive autoregulation, they do not 
establish whether this autoregulatory loop is direct or indirect. Of note, maintenance of bap during stage 11 (but not its initiation during stage 10) also requires bin activity (Fig. 3, cf. J with I). Therefore, it is possible that, at least during stage 11 , bin and bap maintain each other's expression through a cross-regulatory feedback loop.

\section{bin is required for differentiation of the circular} and longitudinal midgut muscles

The function of bin in visceral mesoderm development was studied by staining bin mutant embryos with a variety of markers including the Ig-domain adhesion molecule Fasciclin III (FasIII), the $\mathrm{G}_{\mathrm{o}} \alpha$ subunit Brokenheart (Bkh), and the Armadillo-repeat protein Vimar, which represent early and specific markers for the trunk visceral mesoderm and developing circular midgut musculature (Fig. 4A,C,E; Patel et al. 1987; Lo and Frasch 1998; Fremion et al. 1999). In bin mutant embryos, none of these three markers are expressed in the trunk visceral mesoderm at any stage of development (Fig. 4B,D,F). The specificity of this phenotype is underscored by the observation that the progenitors and differentiated cells of the heart, in which bin is not expressed, show normal expression of all tested markers in bin mutant embryos (see Fig. 4D for bkh; results with Tin and Eve are not shown). In addition, FasIII expression and tissue morphology can be restored in bin mutant embryos to almost normal levels by expressing wild-type bin in the trunk visceral mesoderm primordia under the control of a bap enhancer (Fig. 4K). The disruptions in the trunk visceral mesoderm in bin mutant embryos are slightly stronger than those found in bap null mutants, which show residual FasIII expression in thoracic segments and trace amounts in abdominal segments (coinciding with residual bin expression in these areas; see Fig. 3E) (Fig. 4M). The observed partial rescue of FasIII expression in abdominal segments of bap null mutants upon forced bin expression with a bap enhancer (Fig. 4N, arrows) suggests that bin is a major effector of bap activity in trunk visceral mesoderm development.

The development of the caudal visceral mesoderm in bin mutant embryos was examined using the expression of the FoxC gene crocodile (croc; Häcker et al. 1995) and the bHLH54F gene (Georgias et al. 1997) as markers. The normal expression of both genes in the early caudal visceral mesoderm in bin mutants shows that bin is not required for the formation of these cells. However, in bin mutants, anterior migration of these cells stalls during stage 11 (Fig. 4, cf. $\mathrm{H}$ and J with $\mathrm{G}$ and I). Because bin is expressed in the longitudinal gut muscle precursors (Fig. $2 \mathrm{~F}-\mathrm{K})$, the observed disruption of the migration and differentiation of these cells could reflect a cell-autonomous function of bin. However, the requirement of bin for normal trunk visceral mesoderm differentiation and the known function of the trunk visceral mesoderm in guiding caudal visceral mesoderm migration (Kusch and Reuter 1999; M. Frasch, unpubl.) could also suggest that the observed phenotype is nonautonomous. To distinguish between these two possibilities, we examined
bHLH54F expression in bin mutant embryos in which bin expression was brought back exclusively in the trunk visceral mesoderm via a bap enhancer. In these embryos, bHLH54F expression as well as the migration and morphology of the longitudinal muscle precursors appears normal, thus indicating that the disruption of these processes in the complete absence of bin activity is, at least predominantly, a nonautonomous effect (Fig. 4L). Presumably, the abnormal or absent trunk visceral mesoderm in bin mutants is unable to support proper guidance and differentiation of longitudinal gut muscle precursors. A similar effect is observed for the endoderm, which has also been shown to require normal trunk visceral mesoderm as a substrate for migration (data not shown; Azpiazu and Frasch 1993; Reuter et al. 1993). In summary, the bin phenotypes in the trunk and caudal visceral mesoderm as well as the endoderm are almost identical to the ones that we had observed in tin and bap null mutant embryos. In contrast to the midgut musculature, we did not detect any abnormalities in the morphology or gene expression patterns of the foregut and hindgut visceral mesoderm in either bin or bap mutants (Fig. 4F; data not shown).

Mutation and ectopic expression of bin causes transformations between visceral and somatic mesodermal cell fates

The fate of the primordial cells of the trunk visceral mesoderm in bin or bap mutant embryos was further studied with a bap-lacZ marker (bap3-1acZ; see Materials and Methods). In wild-type as well as bin and bap mutant backgrounds, bap3-lac $Z$ expression initiates during stage 10 in a normal metameric pattern (H.H. Lee and $M$. Frasch, unpubl.), and $\beta$-gal protein perdures in descendants of these cells until late embryogenesis. Therefore, bap-driven $\beta$-gal marks the trunk visceral mesoderm and, at late stages, the circular gut muscle cells of wild-type embryos (Fig. 5A-C). In bin mutant embryos carrying bap3-lac $Z, \beta$-gal stainings show that the presumptive trunk visceral mesoderm primordia segregate towards the interior and coalesce into a band as in wildtype embryos (Fig. 5D). However, there are irregularities in the arrangement of the visceral mesoderm cells, which become much more pronounced after stage 11. At stage 13 , these cells fail to become columnar, are not tightly attached to the endoderm, and become clustered segmentally instead of maintaining a continuous band (Fig. 5, cf. E with B). During stages 14-17, only a few of the bap3-1ac $Z$ expressing cells are attached to the endoderm, whereas the majority of them are scattered in areas within or underneath the somatic mesoderm (Fig. 5, cf. F with C). In addition, the midgut fails to undergo any constrictions. bap null mutants show a similar although more severe phenotype. More specifically, the presumptive visceral mesoderm cells internalize, but coalescence of the clusters during stage 11 is incomplete (Fig. 5G), and at later stages, very few of the bap3-lac Z expressing cells are attached to the endoderm (Fig. 5H,I).

Because of the apparent intermingling of bap3-lacZ- 
Zaffran et al.

Figure 4. bin function is necessary for the development of the midgut visceral mesoderm. $(A, C, E, G, I)$ Wild-type; $(B, D, F, H, J)$ bin mutant embryos. $(K, L, N)$ bin and bap mutant embryos, respectively, with bin expression in the visceral mesoderm provided via bap3GAL4/UAS-bin. (A) Wild-type embryo stained for Fasciclin III, which marks trunk visceral mesoderm (TVM; arrowhead). (B) bin mutant embryo lacking Fasciclin III expression in the area of the TVM. (C) Stage 13 wildtype embryo hybridized with $b k h$ probe. $b k h$ mRNA is expressed in the dorsal vessel, ventral nerve cord, and midgut visceral mesoderm (arrow). (D) bin mutant embryo with $b k h$ expression missing in the area where circular muscle precursors are normally found. (E) Wildtype embryo at late stage 13 stained for vimar-lacZdriven $\beta$-gal expression in midgut (arrow) and hindgut visceral muscle precursors. $(F)$ In bin mutant embryo carrying vimar-lac $Z, \beta$-gal expression is observed in hindgut but not midgut visceral muscles. $(G)$ Late stage 11 embryo stained for $c r o c-l a c Z$ to visualize longitudinal gut muscle precursors (arrowhead). (H) In bin mutant background, croc-lacZ-expressing cells appear normal at stage 11 (arrowhead). (I) Stage 13 embryo hybridized with $b H L H 54 F$ probe to visualize longitudinal muscle precursors after migration. $(J)$ bin mutant embryo stained for $b H L H 54 F$ reveals a strong reduction in the number of longitudinal muscle precursors (arrow, cf. $I$ ). (K) Stage 12 bin mutant embryo with bap-GAL4 driven UAS-bin and stained for Fasciclin III. The circular midgut muscle precursors are rescued, except for a few gaps (arrow). (L) bHLH54F expression in embryo of the same genetic background as in $K$, showing complete rescue of longitudinal gut muscle formation (cf. $J)$. (M) Stage 12 bap null mutant embryo (tinRe28-58,Df(3R) $e^{D 7}$ ) stained for FasIII (arrow, residual FasIII in anterior TVM). (N) bap null embryo (tinRe28-58,Df(3R)e $e^{D 7} / \mathrm{Df}(3 \mathrm{R}) \mathrm{e}^{\mathrm{F1}}$ ) with bap3-Gal4 and UAS-bin (arrows, rescued FasIII in TVM).
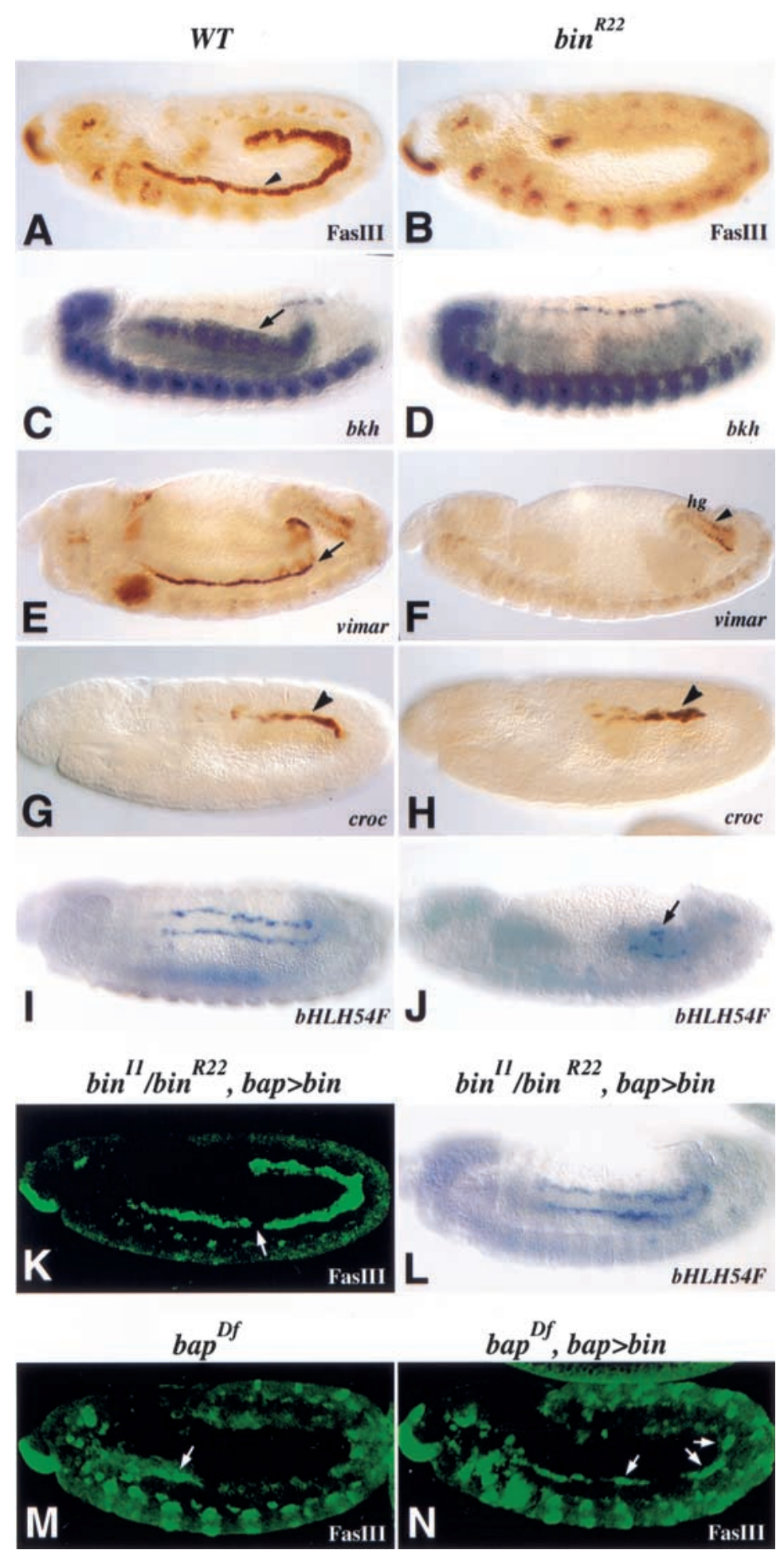

positive cells with somatic mesoderm, we tested whether cells derived from the trunk visceral mesoderm primordia become incorporated into body wall muscle fibers in bin and bap mutant embryos. As shown in Figure 5L-O, syncytial muscle fibers stained for myosin heavy chain (MHC) are positive for $\beta$-gal, whereas in wild-type backgrounds, bap3-lacZ does not produce any significant signals in the somatic musculature (see Fig. $5 \mathrm{~J}, \mathrm{~K})$. Because the particular $\beta$-gal from this construct is both nuclear and cytoplasmic, we infer that the $\beta$-galpositive nuclei seen in the syncytial muscle fibers in bin and bap mutant embryos (Fig. 5L-O, arrowheads) are the ones that are derived from cells that would normally form midgut muscles. In bin mutant embryos, the trans- 


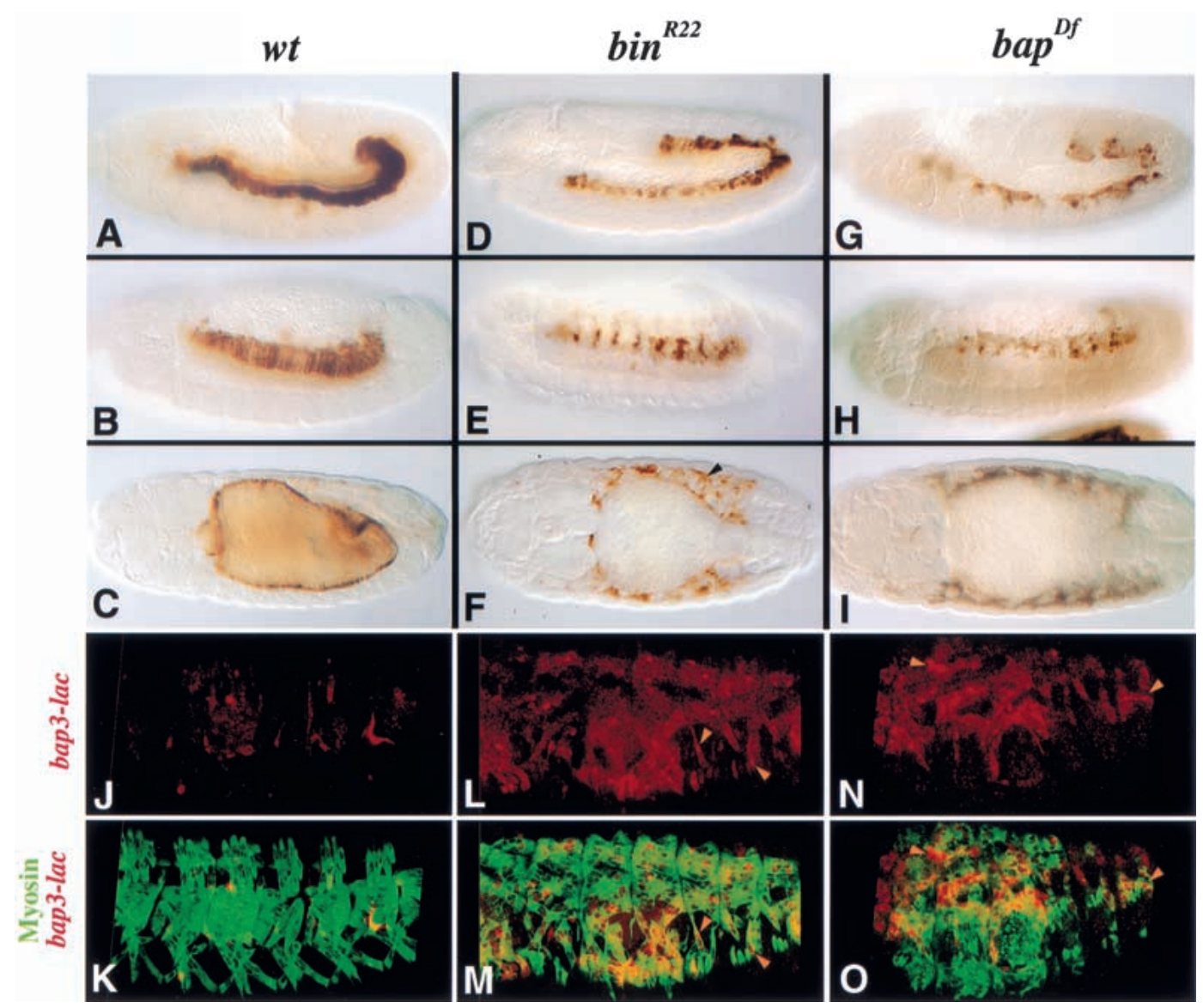

Figure 5. Functions of bin and bap in the maintenance of visceral mesodermal cell fates. Embryos with wild-type $(A-C, J, K), b i n$ mutant $(D-F, L, M)$, and bap null mutant $(G-I, N, O)$ backgrounds carrying a bap3-lacZ insertion. $(A-I)$ Embryos were stained for $\beta$-gal to visualize circular midgut muscle precursors and follow their cell fate. (J-O) Embryos doubly stained for MHC (green) and $\beta$-gal (red) and scanned via confocal microscopy. $(A-C)$ Stage 12,13 , and 15 wild-type embryos carrying bap3-lacZ. $\beta$-Gal from the trunk visceral mesoderm primordia perdures in developing circular midgut muscles. $(D)$ Stage 12 bin mutant embryo in which lacZ-expressing precursors of circular midgut muscles in the dorsal mesoderm are slightly disorganized. (E) Stage 13 bin mutant embryo with segmentally arranged $\beta$-gal-expressing cells in the trunk region. $(F)$ Dorsal view of stage 15 bin mutant embryo. Only a few $\beta$-galstained cells remain around the midgut, whereas the majority of them are localized within or near the body wall muscles layer (arrowhead). $(G, H, I)$ Stage 12, stage 13, and stage 15 bap mutant embryos, respectively, carrying bap3-lacZ. $\beta$-Gal staining reveals visceral mesoderm abnormalities similar to those in bin mutants. $(J, K)$ Stage 16 wild-type embryo with bap-lacZ, doubly stained for MHC (green) and $\beta$-gal (red) proteins. $\beta$-gal protein is rarely detected in somatic muscle cells. $(L, M)$ In bin mutant embryo with bap3-lac $Z$, detection of $\mathrm{MHC}$ (green) and $\beta$-gal (red) proteins shows that cells originating from trunk visceral mesoderm primordia have fused into syncytia of somatic muscles (arrowheads). ( $N, O)$ In bap mutant embryos with bap3-lacZ, MHC (green) and $\beta$-gal (red) proteins are extensively colocalized (arrowheads).

formation from a visceral into a somatic muscle fate appears to affect a large proportion although not all of the bap3-lacZ-positive cells, whereas in bap null mutants, essentially all of them appear to be transformed.

To test whether bin has the potential to cause cell fate transformations from somatic to visceral mesoderm, we assessed the effects of ectopic expression of bin in the entire early mesoderm under the control of a twist enhancer. Ectopic bin causes ectopic expression of bap, which becomes expressed almost uniformly in the dorsal mesoderm (Fig. 6, cf. B with A). The expression of additional differentiation markers for trunk visceral mesoderm, FasIII (Fig. 6C,D), vimar mRNA (Fig. 6E,F), and vimar-lac $Z$ (data not shown), is also expanded upon ectopic bin expression to include ventral areas that are normally occupied by the somatic mesoderm. Stainings for Even-skipped (Eve) demonstrate that ectopic bin interferes with the specification of dorsal somatic muscle and pericardial cell progenitors (Fig. 6, cf. H with G). Furthermore, stainings for $\beta 3$-tubulin and muscle myosin show that ectopic bin disrupts somatic muscle differentiation, including myoblast fusion (Fig. 6, cf. J and L with I and K). Altogether, these observations strongly suggest that the presence of bin activity is able to force the development of somatic mesodermal cells towards the trunk visceral mesoderm pathway. However, the atypical morphology, the supernumerary cells which express visceral mesoderm markers, and the diminished ectopic marker expression at late stages (data not shown) indicate that, at least under these particular conditions 


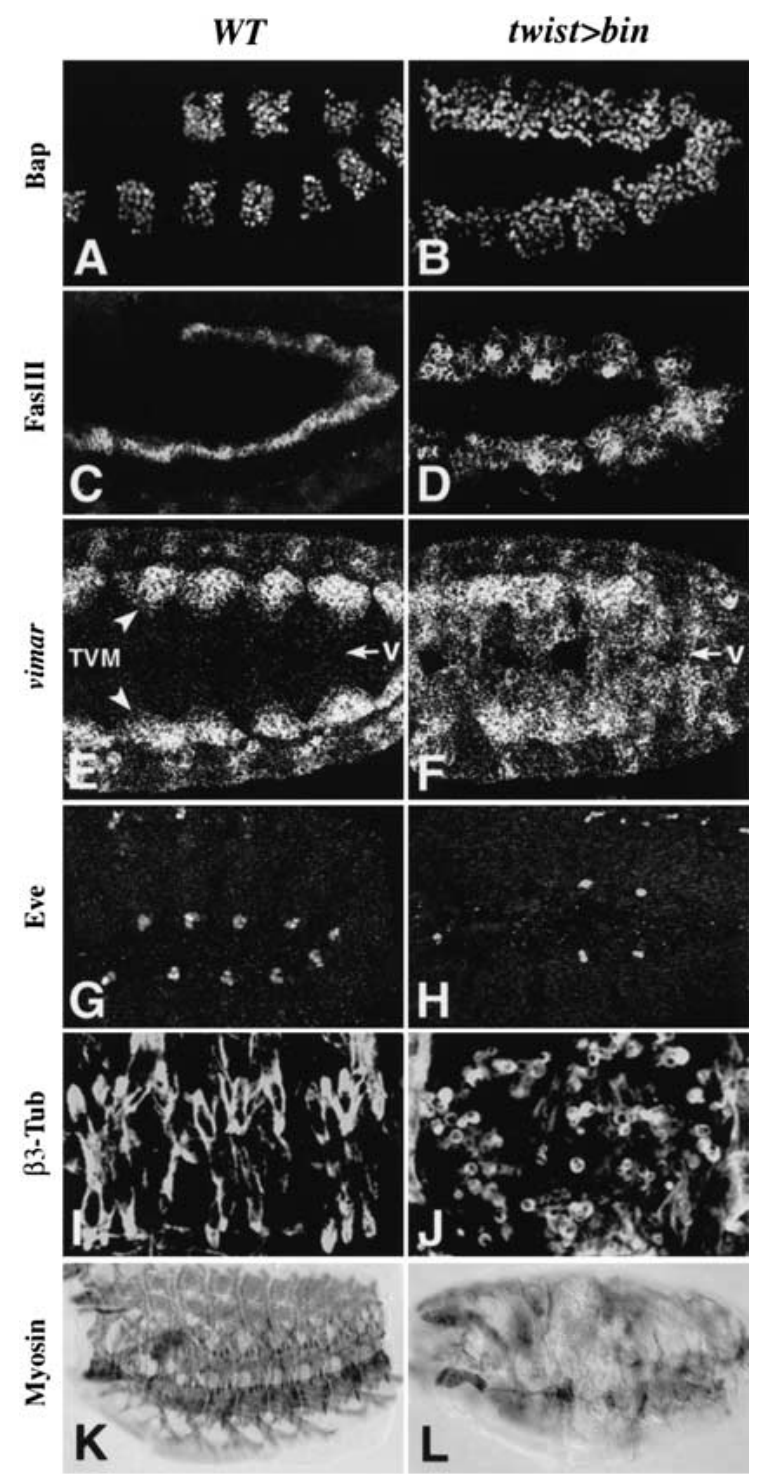

Figure 6. Expansion of visceral mesoderm and disruption of somatic mesoderm as a consequence of bin misexpression. High-magnification views of wild-type (left) and twist-GAL4/ UAS-bin embryos (right). (A) Stage 10 embryo showing Bap protein expression. $(B)$ Upon misexpression of bin in the entire mesoderm, Bap becomes uniformly expressed in the dorsal mesoderm. $(C, D)$ Stage 12 embryos stained for Fasciclin III (FasIII) protein. The wild-type embryo shows FasIII in a narrow band of visceral mesoderm, while the embryo with ectopic bin shows a strong expansion of FasIII expression. $(E, F)$ Early stage $12 \mathrm{em}-$ bryos showing vimar mRNA in the trunk visceral mesoderm (TVM) and, upon ectopic expression of bin, also in ventral areas (v, arrows) normally occupied by somatic musculature. $(G, H)$ Stage 12 embryos stained for Even-skipped (Eve) protein. The embryo with ectopic bin expression shows a reduction of Evepositive pericardial and dorsal muscle progenitors. $(I, J)$ Stage 16 embryos stained for $\beta 3$-Tubulin ( $\beta 3$-Tub) protein, which is expressed in all myogenic cells. The wild-type embryo shows $\beta 3$ Tub in multinucleate somatic muscle fibers, while the embryo with ectopic bin shows mostly unfused, mononucleated cells. $(K, L)$ MHC antibody stainings show a severe reduction of somatic muscle fiber formation upon ectopic bin expression at earlier stages. of ectopic bin expression, the cell fate transformation in this direction are not complete.

bin is a direct upstream regulator of dpp within the visceral mesoderm

Besides tissue-specific differentiation genes that are expressed throughout the trunk visceral mesoderm, several key regulators of midgut morphogenesis are known to be expressed in a spatially restricted manner within this tissue. This type of gene products includes the homeotic factor Ubx and the secreted factor Dpp, both of which are expressed in PS7 of the visceral mesoderm (see Fig. 8E for dpp mRNA, which has an additional expression domain in the prospective gastric caecae in PS3). Although it has been established that Ubx and Dpp maintain their expression in PS7 through a crossregulatory loop and the action of Wg from the adjacent PS8, there is evidence that their expression requires at least one additional, visceral mesoderm-specific cofactor, for which Bin may be a candidate (Panganiban et al. 1990; Reuter et al. 1990; Hursh et al. 1993; Thüringer et al. 1993; Capovilla et al. 1994; Manak et al. 1994; Sun et al. 1995; Yu et al. 1996). To test this possibility, we examined Ubx and $d p p$ expression in bin mutant embryos, which carried bap3lac $Z$ to allow the unambiguous identification of the disrupted visceral mesoderm layer. Visceral mesoderm expression of Ubx in bin mutant embryos is similar to that of wild-type embryos until at least stage 13, although there is a low level of ectopic expression (Fig. 7A,B). Likewise, Ubx expression is also observed in $\beta$-gal-positive cells in bap mutant embryos, albeit with reduced levels and an expanded domain that are comparable to the somatic mesoderm (Fig. 7C). These data demonstrate that the establishment of Ubx expression in the visceral mesoderm requires neither bin nor bap activity. In contrast, $d p p$ is not expressed at any stage in PS7 in the visceral mesoderm of bin mutant embryos, indicating that Bin may serve as a critical tissue-specific cofactor for the regulation of $d p p$ expression (Fig. 7, cf. E with D). The expression of $\mathrm{wg}$ in PS8 is also absolutely dependent on bin activity (data not shown). The absence of these morphogenetic factors is likely to contribute to the defective midgut morphology in bin mutant embryos.

The identification of visceral mesoderm-specific enhancer elements of $d p p$ (Hursh et al. 1993; Manak et al. 1994; Sun et al. 1995) allowed us to test the possibility that bin might be a direct upstream regulator of $d p p$ in the visceral mesoderm. We focused our attention on two minimal enhancer elements, the 130 bp element BM (Sun et al. 1995) and the 231 bp element PB (a slightly shorter version of dpp261 (Manak et al. 1994; see Materials and Methods) (Fig. 8A). PB is able to drive reporter gene expression in PS3 and PS7 of the visceral mesoderm in a pattern that is similar to that of endogenous $d p p$, although PB-lacZ expression in PS7 is less robust (Fig. $8 \mathrm{~F}$; note that unlike the related $d p p 261-1 a c Z$, our construct is not subject to derepression in PS4-6). In contrast to PB and as reported by Sun et al. (1995), BM is active in a broad region extending from PS7 to PS12 in 


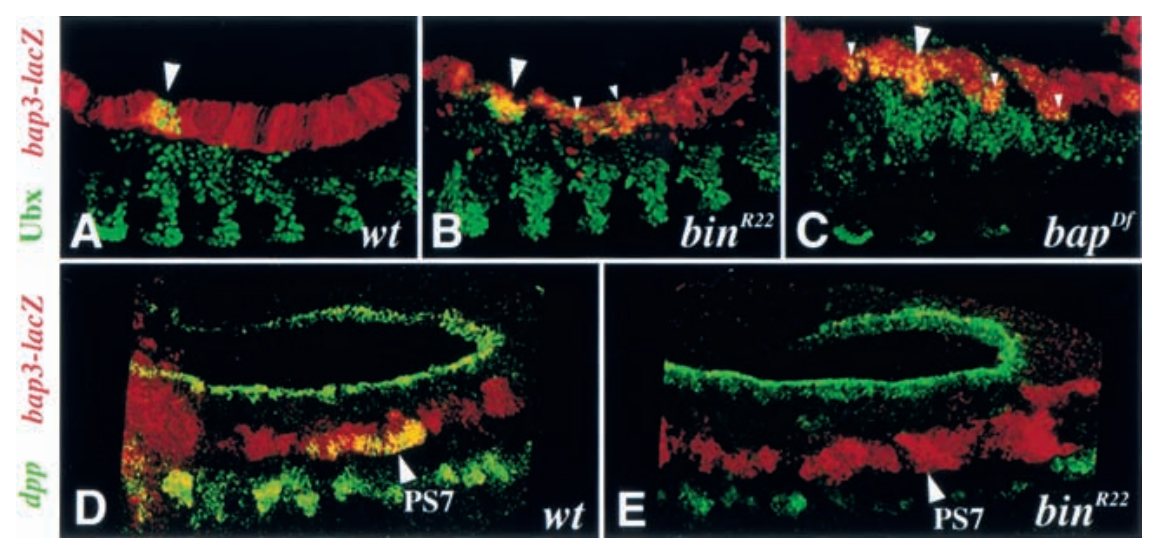

Figure 7. biniou is required for the activation of $d p p$ but not Ubx in PS7 of the midgut visceral mesoderm. Shown are wild-type and mutant embryos carrying bap3-lacZ to identify the visceral mesoderm layer. $(A-C)$ Stage 13-14 embryos double-stained for Ubx (green) and $\beta$-gal (red) proteins. Wild-type $(A)$, bin mutant $(B)$, and bap null mutant $(C)$ embryos all show accumulation of Ubx in PS7 of the visceral mesoderm (large arrowheads; yellow signal). Small arrowheads denote ectopic expression. $(D, E)$ Stage 12 embryos double labeled for $d p p$ mRNA (green) and $\beta$-gal protein (red). In contrast to the wildtype embryo $(D)$, the bin mutant embryo (E) shows a complete lack of $d p p$ mRNA in parasegment 7 (PS7) (arrowheads). the visceral mesoderm (Fig. $8 \mathrm{H}$ ). In addition, the combination of $\mathrm{BM}$ and $\mathrm{PB}$ results in a significant enhancement of PS7 expression compared to PB alone (Fig. 8J; see also Sun et al. 1995). Because of the broad activity of BM in the visceral mesoderm and its enhancing effect on $\mathrm{PB}$ (or longer versions thereof), BM has been proposed to act as a general visceral mesoderm enhancer (GVME), whereas $\mathrm{PB}$ is predominantly targeted by spatially restricted activities that include Ubx and Exd (Fig. 8A; Manak et al. 1994; Sun et al. 1995).

We performed DNaseI protection assays with recombinant Bin protein to test for the presence of Bin binding sites within $\mathrm{BM}$ and $\mathrm{PB}$. These experiments identified two protected regions within BM, termed Bin I and Bin II, which are about 50 bp apart from one another (Fig. 8B). $\mathrm{PB}$ contains a third strongly protected sequence, Bin III, and two minor binding sites which overlap with the previously described Exd binding sites e1 and e2 (Fig. 8C; see Sun et al. 1995). All three of the strongly protected sequences and the weaker el contain sequence motifs that perfectly match forkhead domain binding sites, including the optimal binding site of a vertebrate ortholog, HFH-8 (Fig. 8B-D; Kaufmann et al. 1995; Peterson et al. 1997). The presence of overlapping inverted and direct repeats of this sequence motif in Bin II and Bin III, respectively, may indicate that these two sites represent dimeric binding sites. Interestingly, the sequences of the three strong and two weak Bin binding sites within PB are highly conserved between $D$. melanogaster and $D$. virilis (Manak et al. 1994), suggesting that they are functionally important.

To test whether any of the strong Bin binding sites are required for enhancer activity in vivo, we introduced nucleotide exchanges that completely abolished in vitro binding of Bin (Fig. 8B,C). Mutation of Bin III results in an almost complete loss of PB enhancer activity in PS7 (Fig. 8, cf. G with F), suggesting that Bin binding to Bin III plays an important role for the activation of $d p p$ in this parasegment. The presence of two weak Bin binding sites in the mutated PB derivative may allow residual expression in a few visceral mesoderm cells within PS7 (Fig. $8 \mathrm{G}$, arrowhead). The fact that PS3 expression is not af- fected significantly upon Bin III mutation may be due to the activity of Exd binding sites, of which one was previously shown to regulate PS3 expression (Manak et al. 1994).

BM enhancer activity in the visceral mesoderm is completely lost when both Bin I and Bin II are mutated (Fig. 8, cf. I with H). When this mutated version of BM is combined with a wild-type version of $\mathrm{PB}$, there is no enhancement of PS7 expression and the same pattern as with $\mathrm{PB}$ alone is observed (data not shown; see Fig. 8F). Finally, the combination of BM and $\mathrm{PB}$ with mutated Bin I, II, and III binding sites does not exhibit any significant enhancer activity in PS7 (Fig. 8, cf. K with the wild-type combination in J). These data suggest that both BM and $\mathrm{PB}$ contain functionally important Bin binding sites. Bin binding to Bin I and Bin II may be key to providing BM with its general visceral mesoderm enhancer activity, whereas binding to Bin III is required in concert with spatially restricted activities to provide the $\mathrm{PB}$ enhancer with a basal level of activity in PS7.

\section{Discussion}

Despite their diverse developmental origins, the different types of visceral muscles in Drosophila share a number of morphological and functional characteristics, which clearly distinguish them from the body wall and cardiac musculature. By analogy to the situation in vertebrate skeletal muscles, one might expect that these common features are due to the activity of one or several key regulatory factors that are shared among the developing circular and longitudinal midgut muscles as well as foregut and hindgut muscles. In the present study, we identified the forkhead domain gene biniou (bin) as a regulator of visceral muscle identities in Drosophila. bin represents the first example of a gene that is specifically expressed in all types of visceral muscles and their precursors. Our analysis focused on the regulation and function of bin in the developing trunk visceral (or splanchnic) mesoderm and the derived circular midgut musculature. 
Zaffran et al.

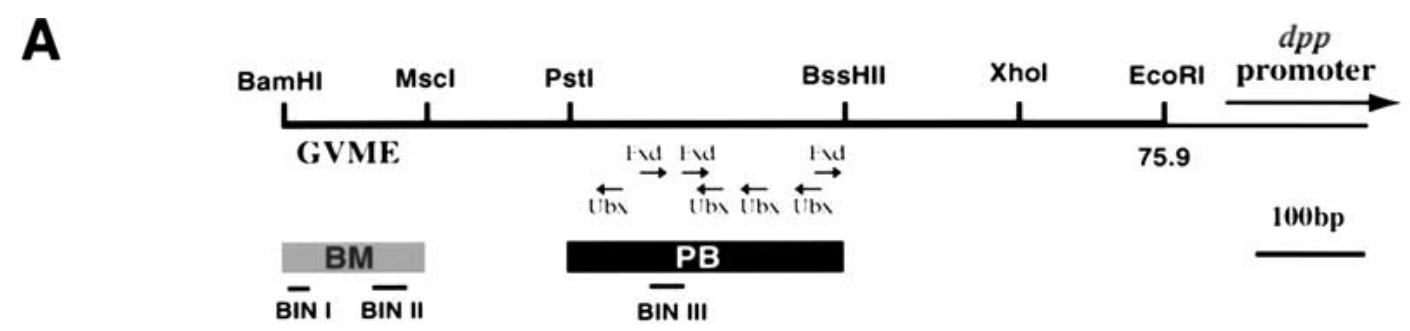

B
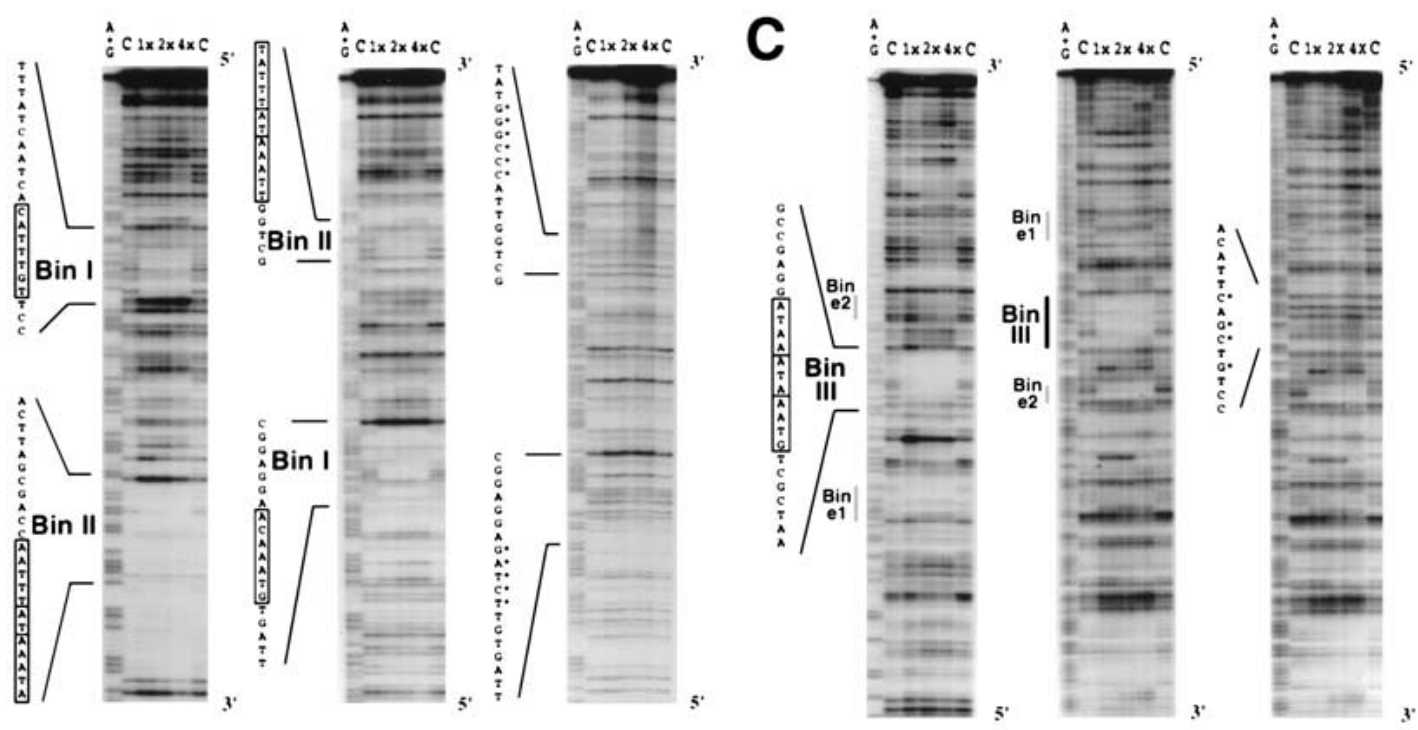
D Bin I (16.29)
Bin II $\quad(87-100)$
Bin II. (102.89)
$\begin{array}{ll}\text { Bin III } & (387.400) \\ \text { Bin III. } & (387.405)\end{array}$
$\mathrm{HFH} \cdot 8$ consensus

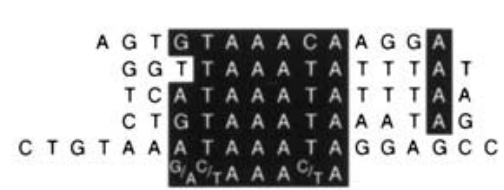

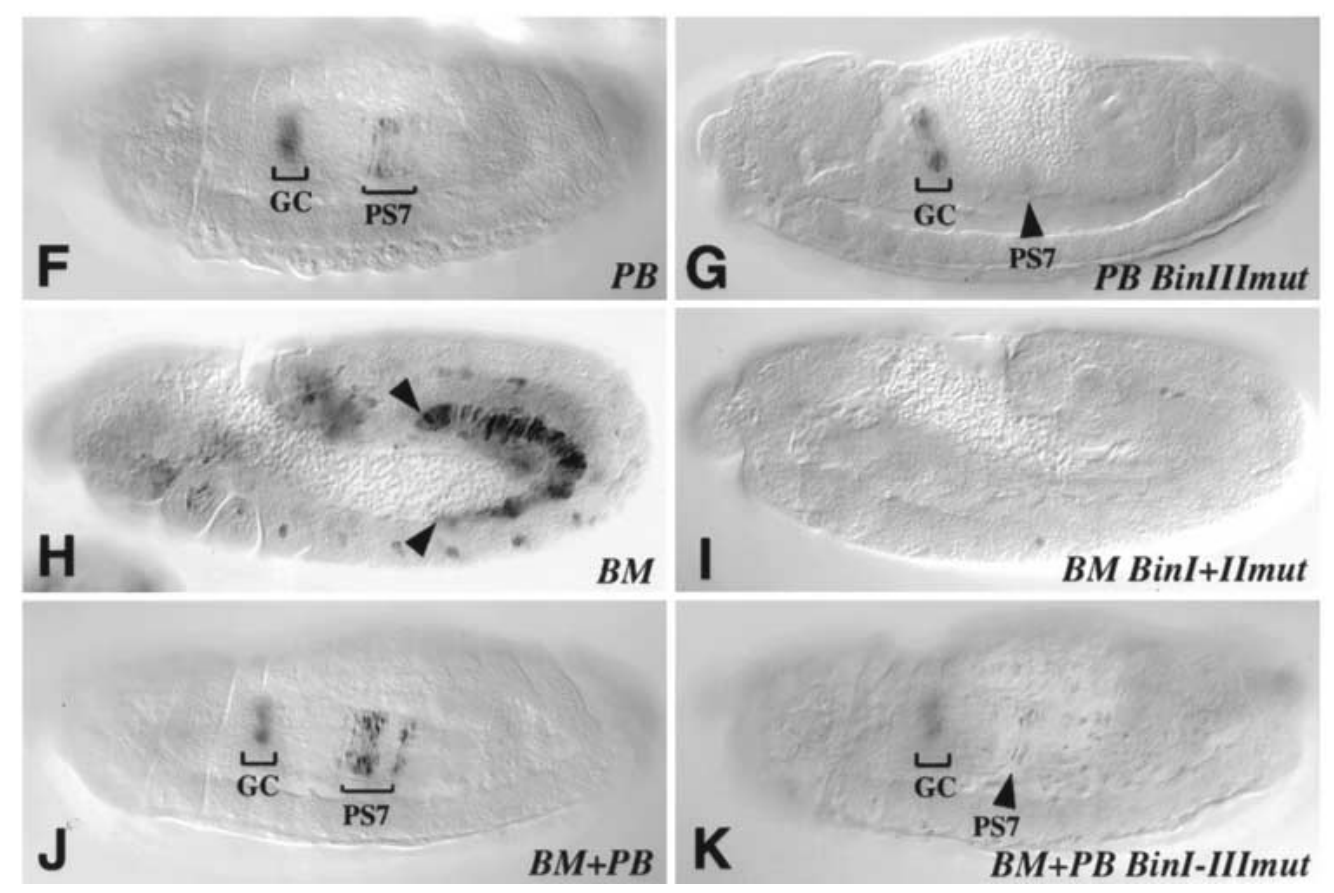

Figure 8. (Legend on facing page) 
bin as a component of a regulatory gene network during trunk visceral mesoderm development

Previous studies established bap as a key regulator in the trunk visceral mesoderm primordia and showed that the spatial domains of bap expression are defined by intersecting dorsoventral and anteroposterior cues (Azpiazu and Frasch 1993; Azpiazu et al. 1996). It was determined that the combined activities of the homeobox gene tin and ectodermally derived Dpp activate bap in the dorsal mesoderm, while $w g$ via its downstream gene $s l p$ causes an abrogation of this activation process (StaehlingHampton et al. 1994; Frasch 1995; Riechmann et al. 1997; Lee and Frasch 2000). As a result, bap expression is turned on in a segmentally repeated pattern within the dorsal mesoderm. The data presented herein indicate that bin requires the combined activities of bap and $d p p$ for its normal activation, which suggests that bin is positioned furthest downstream in the known regulatory hierarchy of transcription factors during visceral mesoderm development. Another important conclusion is that inductive Dpp signals are active not just once in the dorsal mesoderm but rather operate successively during each of the three known steps of gene activation that participate in the pathway leading to trunk visceral mesoderm (Fig. 9). These activation steps (tin $\rightarrow$ dorsal tin $\rightarrow$ bap $\rightarrow$ bin) occur in rapid succession, apparently within minutes after the mesoderm has spread beneath the dorsal ectodermal domain of Dpp expression.

We have shown previously that the induction of tin expression by Dpp in the dorsal mesoderm involves the combined binding of Smad proteins (Medea and Mad) and Tin itself to a Dpp-responsive enhancer of the tin gene (Xu et al. 1998). Our genetic data for bin suggest an analogous mechanism for bin, in which the combined binding of Smads and Bap (an NK homeodomain protein related to Tin) may activate a Dpp-responsive bin enhancer. However, in contrast to the induction of tin and bap by Dpp, which stringently requires tin activity, low levels of bin can be induced by Dpp even in the absence of tin and bap. This observation indicates that there is at least one other mesoderm-intrinsic factor, in addition to

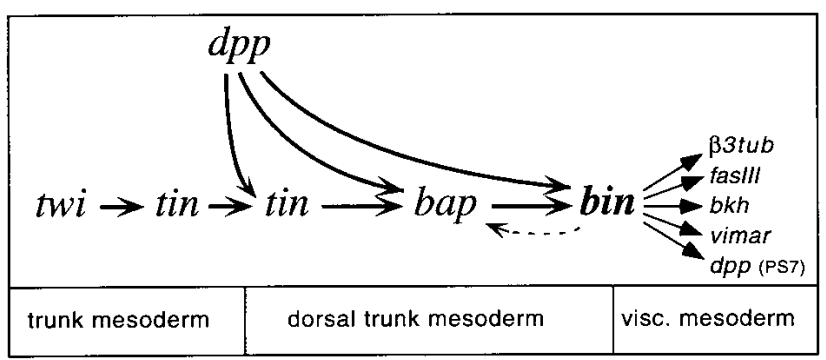

Figure 9. Regulatory interactions during specification and differentiation of the trunk visceral mesoderm. Only positive inputs are shown in the diagram.

Tin and Bap, which helps to provide the mesoderm with the competence to respond to Dpp and allow bin induction.

The relatively normal spatial pattern of residual bin expression in the absence of bap indicates that bin may also receive direct inputs from striped regulators. This regulation could be analogous to that of bap as well and involve the negative activities of $w g$ and slp. Lastly, we have shown that there is feedback regulation in which bin regulates prolonged expression of bap. However, this indirect feedback loop is only operative until early stage 12, when bap expression ceases in the trunk visceral mesoderm. The maintenance of bin expression during later stages of visceral mesoderm development could involve direct autoregulation.

\section{The roles of bin and bap in visceral mesoderm specification and differentiation}

The similarities in the early expression patterns of bin and bap in the trunk visceral mesoderm primordia raise the question of the relative contribution of these two genes to visceral mesoderm development. Are all functions of bap in this developmental process mediated through bin, or does bap also fulfill bin-independent roles? We have shown that in the absence of either bin or

Figure 8. Bin binding sites in $d p p$ enhancers are essential for enhancer activity in parasegment 7 of the visceral mesoderm. $(A) d p p$ VM enhancer region upstream of $d p p$ (from BamHI to EcoRI; see Manak et al. 1994; Sun et al. 1995). (GVME) General visceral mesoderm enhancer (Sun et al. 1995). The PstI/BssHII (PB) fragment refers to the 261-bp enhancer of Manak et al. (1994). Previously identified Ubx and Exd binding sites within PB are shown as in Manak et al. (1994). (B) DNase I footprinting analysis with increasing amounts of Bin protein or no Bin (lane $C$ ) of ${ }^{32} \mathrm{P}$-labeled $d p p$-BM and $(C)$ of $d p p$-PB sequences, respectively. Binding experiments with wild-type sequences (sense and antisense) are shown to the left and experiments with mutated sequences to the right (see asterisks; antisense strand is shown for BM and sense strand for PB). The protected regions (boxed sequences) are named Bin I, II, or III. Bin/e1 and Bin/e2 are sites of weak protection with Bin that also bind Exd (Manak et al. 1994; Sun et al. 1995). (D) Sequence alignments of protected Bin binding sites within $d p p-\mathrm{BM}, d p p-\mathrm{PB}$, and the HFH-8 consensus sequence (Peterson et al. 1997). (Asterisks) Alignments of the second copies of tandemly arranged consensus motifs within Bin II and Bin III. Nucleotide numbers refer to GenBank entry X81977. (E) Stage 14 wild-type embryo stained for $d p p$ mRNA expression for a comparison and $(F-H)$ embryos carrying various $d p p$-lac $Z$ constructs and stained for $\beta$-gal expression. $(F)$ The 231-bp $d p p$-PB element drives expression in PS7 and the gastric caeca primordia (GC). $(G)$ Mutation of the Bin III binding site within PB causes severe reduction of PS7 expression. $(H)$ The 130-bp $d p p$-BM element drives uniform expression starting from stage 11 in the posterior portion (PS7-12) of the visceral mesoderm. (I) Mutation of both Bin I and Bin II binding sites within the dpp-BM element abolishes enhancer activity (cf. $H$ ). (J) Combination of BM with PB produces strongly enhanced PS7 expression (cf. F). $(K)$ Mutation of all three major bin binding sites within the combined BM+PB elements leads to a near loss of PS7 expression (cf. J). 
bap activity, a large portion of the cells that are normally destined to form visceral muscles become incorporated into body wall muscles. Likewise, the expression of several trunk visceral mesoderm markers is affected similarly in bin and bap mutants (Fig. 4; Lo and Frasch 1998). The similarities of these phenotypes as well as the partial rescue of bap phenotypes upon forced expression of bin suggest that most of the activities of bap in the trunk visceral mesoderm involve the activation and function of its downstream gene bin. Because of the temporal overlap of Bap and Bin expression during stage 10 to early stage 12 , it is also possible that both proteins are required in combination to activate some target genes. Based upon our lineage tracing data, the transformation from visceral into somatic muscle fates appears to be more complete in bap mutants compared to bin mutants, and defects in the migration behavior and morphogenesis of the trunk visceral mesoderm are evident at an earlier stage in bap mutants. It is therefore likely that bap has some additional targets in the visceral mesoderm that do not require bin.

The lineage tracing experiments with the bap-lacZ marker also showed that the earliest steps of trunk visceral mesoderm, which consist of the segregation of primordial cells from the mesodermal monolayer towards the interior, do not require bap or bin activity. This behavior is unlikely to be due to functional redundancy between the two genes, because bin expression is largely missing in bap mutants. Rather, this observation points to the existence of regulatory gene(s) in addition to bap and bin that are present in the 11 clusters of dorsal mesodermal cells and control their segregation towards the interior to form visceral mesoderm. The emerging picture is that multiple regulatory genes are activated in metameric clusters of dorsal mesodermal cells that define the trunk visceral mesoderm primordia. Based on the available genetic data, it is likely that the induction of all of these regulators requires Dpp and is prevented by slp. In addition to Dpp, bap is strictly and bin largely dependent on tin, whereas induction of yet unknown genes that regulate visceral mesoderm segregation may be largely or fully independent of tin (Taylor 2000). While each of these regulatory genes appears to have some unique functions in visceral mesoderm development, we have shown herein that three of the Dpp-induced genes, tin, bap, and bin, are part of a mesodermintrinsic cascade of gene activations, which also involves feedback regulation. We propose that a major outcome of this regulatory cascade is the activation of bin in the primordial cells of the trunk visceral mesoderm and its maintenance in the developing circular midgut muscles. It appears that bin plays a key role in activating multiple, if not the majority, of patterning and differentiation genes which define the morphological and functional features of midgut muscles and prevent visceral mesodermal cells from fusing with somatic muscle precursors. Molecular and genetic studies show that at least two of these downstream genes, $d p p$ (see below) and $\beta$-3tubulin (S. Zaffran and M. Frasch, in prep.), are direct targets of bin.
Bin as a tissue-specific cofactor controlling dpp expression in the visceral mesoderm

Spatially restricted expression of $d p p$ in PS7 of the trunk visceral mesoderm plays an important role in normal midgut morphogenesis (Immerglück et al. 1990; Hursh et al. 1993; Masucci and Hoffmann 1993). Previous studies have identified Ubx and Exd as directly and positively acting upstream regulators of $d p p$ in the visceral mesoderm (Reuter et al. 1990; Hursh et al. 1993; Capovilla et al. 1994; Chan et al. 1994; Manak et al. 1994; Sun et al. 1995). Because these regulators are present in other tissues where they do not activate $d p p$ expression, it has been proposed that they require tissue-specific cofactor(s) for activating $d p p$ in the visceral mesoderm (Hursh et al. 1993). Further evidence of the involvement of cofactor(s) that are predicted to be expressed uniformly in the visceral mesoderm came from the dissection of the visceral mesodermal $d p p$ enhancer. In particular, truncations or specific mutations within this enhancer caused an expansion of enhancer activity beyond the Ubx domain and in some cases throughout the trunk visceral mesoderm (Manak et al. 1994; Sun et al. 1995; Yang et al. 2000). These data suggest that, in the absence of repressing activities, uniformly expressed visceral mesodermal factor(s) are able to activate the $d p p$ enhancer without a requirement for Ubx and Exd.

The present study demonstrates that the FoxF protein Bin corresponds to such a visceral mesoderm-specific factor during the activation of $d p p$ expression. Bin is expressed in the visceral mesoderm prior to $d p p$, is genetically required for $d p p$ expression, and the Bin binding sites within the $d p p$ enhancer are essential for enhancer activity in PS7 of the visceral mesoderm. Of note, our data suggest that Bin has a key role in the activation of a previously identified general visceral mesoderm enhancer of $d p p$ (see Fig. 8; Sun et al. 1995), which is active throughout PS7 to PS12 of the visceral mesoderm. In the normal context, Bin binding to these sequences is required for the enhancement of $d p p$ expression in PS7. However, the presence of additional functionally important Bin binding sites that are interdigitated with Ubx and Exd sites demonstrates that Bin is an integral component of PS7-specific enhancer activation as well and that a clean separation between general and PS7-specific enhancer elements does not exist. The available data suggest that PS7-specific expression of $d p p$ in the visceral mesoderm is regulated by an exquisite balance between positive and negative activities. Negative regulators have been shown to include dTCF (or a factor with related binding specificity), which acts in a wg-independent manner in the entire trunk visceral mesoderm (Yang et al. 2000), and Abd-A, which is only active between PS8 and PS12 (Reuter et al. 1990). Based on these combined data, we propose a model in which the activator bin is neutralized by the negative factors to provide a sensitized equilibrium of gene activities, which is set below the threshold level of $d p p$ activation. In this model, the role of Ubx and Exd would be to disrupt this equilibrium and shift it towards the active state in PS7. 
Evolutionary conservation of FoxF gene functions in the splanchnic mesoderm?

An early binary decision during mesoderm development in both insect and vertebrate embryos results in the splitting of a single layer into the two separate layers of somatic and splanchnic mesoderm, which become associated with lateral ectoderm and endoderm, respectively. In Drosophila and other insects, the splanchnic mesoderm develops exclusively into gut musculature, whereas in vertebrates it contributes to many additional internal organs. Interestingly, the observations described in the present paper together with recently published data suggest that the overt morphological and developmental similarities in the splanchnic mesoderm of insects and vertebrates extend to the molecular level. Similar to Drosophila bin, the two vertebrate orthologs FoxF1 and FoxF2 (previously termed FREAC1/2 [mouse, rat, human Foxf1/2], $H F H-8$ [mouse Foxf1], lun [mouse Foxf2], and XFD-13 [frog FoxF1]) are predominantly expressed in the splanchnic mesoderm and mesenchyme that line the digestive tract (Hellqvist et al. 1996; Peterson et al. 1997; Mahlapuu et al. 1998; Funayama et al. 1999; Köster et al. 1999; Aitola et al. 2000). At later stages, expression continues in various tissues that are derived from splanchnic mesoderm, including smooth muscles of the intestine, lung, and liver capsule. In addition to similarities in their expression patterns, the phenotype of mouse embryos with a targeted disruption of Foxf1 suggests similarities in developmental functions of the Drosophila and vertebrate genes. In Foxf1-/embryos, splitting of the lateral plate mesoderm into splanchnic and somatic layers is frequently incomplete or absent, and ectopic expression of the somatic mesoderm marker Irx1 indicates that the splanchnic mesoderm assumes characteristics of somatic mesoderm (Mahlapuu et al. 2001). Both of these alterations are strongly reminiscent of the bin phenotype. Other striking similarities with Drosophila bin include the requirement of Foxf1 for the activation of BMP4 expression in the lateral plate and allantois (Mahlapuu et al. 2001) as well as its coexpression with the bap ortholog Bapx1 in the splanchnic mesoderm (Tribioli et al. 1997). Moreover, the demonstration that the lateral plate mesoderm is under the influence of BMP signaling (Tonegawa et al. 1997) could imply that, analogous to bin, which is initially a target of Dpp and at later stages activates $d p p$ expression, FoxF genes are both downstream and upstream of BMPs. Collectively, these data suggest that the splanchnic mesoderm in insect and vertebrate embryos are homologous tissues and that their development is controlled by related genetic circuits in which members of the FoxF class of forkhead domain genes occupy a central position.

\section{Materials and methods}

Drosophila strains and genetics

The genotype termed $b a p^{D f}$, which was obtained by crossing $D f(3 R) e^{F 1}$ and a $D f(3 R) e^{D 7}$ chromosome carrying the tin geno- mic rescue construct tin Re28-58 (Azpiazu and Frasch 1993), produces an amorphic bap phenotype in the visceral mesoderm that can be rescued by adding a bap Re3 genomic rescue construct (M. Frasch, unpubl.). The bap3-lacZ \#6 line carries a bap enhancer/pCaSpeRhs43 $\beta$-gal construct on the second chromosome (H.-H. Lee and M. Frasch, unpubl.). The null allele $t_{i n}^{346}$ and hypomorphic allele bap $^{208}$ were described by Azpiazu and Frasch (1993), the croc-lacZ line $\operatorname{croc}^{5 F 59}$ by Häcker et al. (1995), and B6-2-30 (vimar-lacZ) by Lo and Frasch (1998). The EMS alleles $\operatorname{bin}^{I 1}$, $\operatorname{bin}^{R 22}$, and $\operatorname{bin}^{S 4}$ were isolated in an $\mathrm{F}_{2}$ screen using the deficiency $D f(3 L) W 5.4$ (Anderson et al. 1995); the collection of lethal mutations at this locus was kindly provided by Wayne Johnson. The phenotypes of homozygous bin alleles were identical to each other and to those of various allelic combinations in trans. bin mutations were balanced over either TM3Sb P[ftz-lacZ] or TM3Sb P[eve-lacZ] to identify homozygous mutant embryos.

\section{Molecular techniques and point mutation detection}

A PCR fragment covering the forkhead domain sequence of bin was isolated by using the P1 clone DS06194 (from BDGP) as a template. The fragment was used to screen a 4- to 8-hr embryonic cDNA library (Brown and Kafatos 1988), and the longest cDNA (2413 bp plus poly A) was sequenced. Genomic DNA was isolated from handpicked homozygous bin mutant embryos, which were stained with a $\beta$-galactosidase antibody to exclude embryos carrying TM3-lacZ chromosomes. DNA fragments corresponding to exons were amplified by PCR and cloned into pCRII-Topo (Invitrogen). All PCR amplifications were repeated several times, and the products were sequenced on both strands. The GST/Bin fusion protein was obtained by cloning a BamHI/ BamHI bin fragment (amino acids 135-615) into pGEX-2T (Pharmacia), and DNaseI footprinting assays were performed as described by Lee and Frasch (2000).

\section{Generation of UAS/GAL4 constructs}

Full-length bin and bap cDNAs were cloned into pUAST (Brand and Perrimon 1993). A UAS-dpp line (Frasch 1995), twist-GAL4 (SG24; Greig and Akam 1993), and bap3-GAL4 were used for ectopic expression studies. For the latter, a 180-bp fragment corresponding to a minimal trunk visceral mesoderm enhancer of bap (H-H. Lee and M. Frasch, unpubl.) was cloned into EcoRI/ NotI of the pGAL4-221 vector (gift from Christian Klämbt, Münster University, Germany).

\section{Construction of dpp-lacZ transformation plasmids}

$d p p$ upstream enhancer elements were obtained by PCR amplification with Drosophila genomic DNA and subcloned into pBluescript KS (Stratagene) or pCRII-Topo (Invitrogen). Based on published sequence information (Manak et al. 1994; Sun et al. 1995), the primers dpp3'-PstI, 5'-GTTCTGTTGCGGGATCC GAAATAGTTAGT-3' and $d p p 3^{\prime}$-NotI, $5^{\prime}$-ATTACTGTCAAG TGGCCAACGGAAGGGAGAGACATC-3' were used to generate $d p p-\mathrm{BM}(+1 /+149)$, and the primers $d p p 5^{\prime} d w, 5^{\prime}-\mathrm{GGC}$ GATTACGTGGAGTACTAC-3' and $d p p 5^{\prime}$ up , 5' -CGATTCAA ATTTATTACTAATTGGGTG-3' were used to generate the dpp-PB $(+282 /+513)$.

For site-directed in vitro mutagenesis of $d p p$ elements, pBluescript KS $d p p-\mathrm{BM}$ and pCRII-Topo $d p p$-PB were used as templates for inverse PCR. Appropriate oligonucleotides were designed to introduce restriction sites replacing the forkhead domain binding sites. The primers 5 '-AGTGTCTAGAGGAGG CACTCTTGAGAACG-3' and 5'-TCCTCTAGAACACTAACT 
ATTTCGGATCCC-3', which introduce an XbaI site, and 5'-

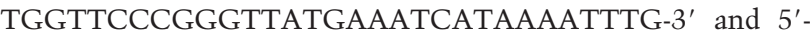
ATTTCATACCCGGGTAACCAGCGATTCAATC-3' , which introduce an SmaI site, were used to mutate Bin I and Bin II, respectively, in $d p p$-BM. The primers 5 -GTAAGTCGACAG GAGCCGCAGATCAAAGG-3' and 5'-CCTGTCGACTTACG CGATTGTAAAACAA-3' were used to mutate Bin III within $d p p-\mathrm{PB}$. All constructs were sequenced, then cloned into pCaSpeRhs43 $\beta$-gal, and six transformant lines were analyzed for each construct.

\section{Embryo staining}

The following primary antibodies were used: rabbit anti- $\beta$-galactosidase (1:3000; Cappel), rabbit anti-Bap and rabbit anti-Eve (1:500 and 1:2000, respectively; Lee and Frasch 2000), rabbit anti- $\beta 3$-Tubulin (1:1500; gift from R. Renkawitz-Pohl, Marburg University, Germany), mouse anti-Fasciclin III (7G10, 1:20; Developmental Studies Hybridoma Bank, Univ. of Iowa), mouse anti-Ubx 1:10; gift from Rob White, Cambridge University, England), mouse anti-myosin heavy chain (1:5; gift from D. Kiehart, Duke University, Durham, NC), and rat anti-Bin (1:500; this work). For Bap and Bin antibody fluorescent stainings, Tyramide Signal Amplification (NEN) in conjunction with the VectaStain $\mathrm{ABC}$ components and microscopy was done as in Zaffran and Frasch (2000). Bin antibodies were raised in rats against a His fusion protein containing amino acids 135-615 of Bin (bin BamHI/BamHI cDNA fragment) induced from a pET30a expression vector (Novagen).

\section{Acknowledgments}

We gratefully acknowledge Wayne Johnson and the Bloomington Stock Collection for fly stocks and the BDGP for sequence information. We thank the Developmental Studies Hybridoma Bank, Dan Kiehart, Renate Renkawitz-Pohl, and Rob White for antibodies and Hanh Nguyen for comments on the manuscript. The confocal laser scanning microscope at the MSSM-Microscopy Center was supported with funding from NIH (1 S10 RR0 9145-01) and NSF (DBI-9724504). We acknowledge the funding of this research by NIH (HD30832 and DK59406).

The publication costs of this article were defrayed in part by payment of page charges. This article must therefore be hereby marked "advertisement" in accordance with 18 USC section 1734 solely to indicate this fact.

\section{References}

Aitola, M., Carlsson, P., Mahlapuu, M., Enerback, S., and PeltoHuikko, M. 2000. Forkhead transcription factor FoxF2 is expressed in mesodermal tissues involved in epithelio-mesenchymal interactions. Dev. Dyn. 218: 136-149.

Anderson, M., Perkins, G., Chittick, P., Shrigley, R., and Johnson, W. 1995. drifter, a Drosophila POU-domain transcription factor, is required for correct differentiation and migration of tracheal cells and midline glia. Genes \& Dev. 9: $123-137$.

Azpiazu, N. and Frasch, M. 1993. tinman and bagpipe: Two homeo box genes that determine cell fates in the dorsal mesoderm of Drosophila. Genes \& Dev. 7: 1325-1340.

Azpiazu, N., Lawrence, P., Vincent, J.-P., and Frasch, M. 1996. Segmentation and specification of the Drosophila mesoderm. Genes \& Dev. 10: 3183-3194.

Bienz, M. 1994. Homeotic genes and positional signalling in the Drosophila viscera. Trends Genet. 10: 22-26.
Bodmer, R. 1993. The gene tinman is required for specification of the heart and visceral muscles in Drosophila. Development 118: 719-729.

Brand, A.H. and Perrimon, N. 1993. Targeted gene expression as a means of altering cell fates and generating dominant phenotypes. Development 118: 401-415.

Brown, N.H. and Kafatos, F.C. 1988. Functional cDNA libraries from Drosophila embryos. J. Mol. Biol. 203: 425-437.

Campos-Ortega, J.A. and Hartenstein, V. 1997. The embryonic development of Drosophila melanogaster. Springer Verlag, Berlin, Germany.

Capovilla, M., Brandt, M., and Botas, J. 1994. Direct regulation of decapentaplegic by Ultrabithorax and its role in Drosophila midgut morphogenesis. Cell 76: 461-475.

Chan, S.K., Jaffe, L., Capovilla, M., Botas, J., and Mann, R.S. 1994. The DNA binding specificity of Ultrabithorax is modulated by cooperative interactions with extradenticle, another homeoprotein. Cell 78: 603-615.

Frasch, M. 1995. Induction of visceral and cardiac mesoderm by ectodermal Dpp in the early Drosophila embryo. Nature 374: 464-467.

Fremion, F., Astier, M., Zaffran, S., Guillen, A., Homburger, V., and Semeriva, M. 1999. The heterotrimeric protein Go is required for the formation of heart epithelium in Drosophila. J. Cell Biol. 145: 1063-1076.

Funayama, N., Sato, Y., Matsumoto, K., Ogura, T., and Takahashi, Y. 1999. Coelom formation: Binary decision of the lateral plate mesoderm is controlled by the ectoderm. Development 126: 4129-4138.

Georgias, C., Wasser, M., and Hinz, U. 1997. A basic-helix-loophelix protein expressed in precursors of Drosophila longitudinal visceral muscles. Mech. Dev. 69: 115-124.

Goldstein, M.A. and Burdette, W.J. 1971. Striated visceral muscle of Drosophila melanogaster. J. Morphol. 134: 315334.

Greig, S. and Akam, M. 1993. Homeotic genes autonomously specify one aspect of pattern in the Drosophila mesoderm. Nature 362: 630-632.

Häcker, U., Kaufmann, E., Hartmann, C., Jürgens, G., Knöchel, W., and Jäckle, H. 1995. The Drosophila fork head domain protein crocodile is required for the establishment of head structures. EMBO J. 14: 5306-5317.

Hellqvist, M., Mahlapuu, M., Samuelsson, L., Enerback, S., and Carlsson, P. 1996. Differential activation of lung-specific genes by two forkhead proteins, FREAC-1 and FREAC-2. I. Biol. Chem. 271: 4482-4490.

Holley, S. and Ferguson, E. 1997. Fish are like flies are like frogs: Conservation of dorsal-ventral patterning mechanisms. BioEssays 19: 281-284.

Hursh, D., Padgett, R., and Gelbart, W. 1993. Cross regulation of decapentaplegic and Ultrabithorax transcription in the embryonic visceral mesoderm of Drosophila. Development 117: $1211-1222$.

Immerglück, K., Lawrence, P., and Bienz, M. 1990. Induction across germ layers in Drosophila mediated by a genetic cascade. Cell 62: 261-268.

Kaufmann, E., Müller, D., and Knöchel, W. 1995. DNA recognition site analysis of Xenopus winged helix proteins. J. Mol. Biol. 239-254.

Köster, M., Dillinger, K., and Knöchel, W. 1999. Genomic structure and embryonic expression of the Xenopus winged helix factors XFD-13/13'. Mech. Dev. 88: 89-93.

Kusch, T. and Reuter, R. 1999. Functions for Drosophila brachyenteron and forkhead in mesoderm specification and cell signalling. Development 126: 3991-4003.

Lee, H. and Frasch, M. 2000. Wingless effects mesoderm 
patterning and ectoderm segmentation events via induction of its downstream target sloppy paired. Development 127: 5497-5508.

Lo, P. and Frasch, M. 1998. bagpipe-dependent expression of vimar, a novel armadillo-repeats gene, in the Drosophila visceral mesoderm. Mech. Dev. 72: 65-75.

Mahlapuu, M., Pelto-Huikko, M., Aitola, M., Enerback, S., and Carlsson, P. 1998. FREAC-1 contains a cell-type-specific transcriptional activation domain and is expressed in epithelial-mesenchymal interfaces. Dev. Biol. 202: 183-195.

Mahlapuu, M., Ormestad, M., Enerback, S., and Carlsson, P. 2001. The forkhead transcription factor Foxf1 is required for differentiation of extra-embryonic and lateral plate mesoderm. Development 128: 155-166.

Manak, J., Mathies, L., and Scott, M. 1994. Regulation of a decapentaplegic midgut enhancer by homeotic proteins. Development 120: 3605-3619.

Masucci, J.D. and Hoffmann, F.M. 1993. Identification of two regions from the Drosophila decapentaplegic gene required for embryonic midgut development and larval viability. Dev. Biol. 159: 276-287.

Panganiban, G.E.F., Reuter, R., Scott, M.P., and Hoffmann, F.M. 1990. A Drosophila growth factor homolog, decapentaplegic, regulates homeotic gene expression within and across germlayers during midgut morphogenesis. Development 110: 1041-1050.

Patel, N.H., Snow, P.M., and Goodman, C.S. 1987. Characterization and cloning of fasciclin III: A glycoprotein expressed on a subset of neurons and axon pathways in Drosophila. Cell 48: 975-988.

Peterson, R., Lim, L., Ye, H., Zhou, H., Overdier, D., and Costa, R. 1997. The winged helix transcriptional activator HFH-8 is expressed in the mesoderm of the primitive streak stage of mouse embryos and its cellular derivatives. Mech. Dev. 69: 53-69.

Reuter, R., Panganiban, G.E.F., Hoffmann, F.M., and Scott, M.P. 1990. Homeotic genes regulate the spatial expression of putative growth factors in the visceral mesoderm of Drosophila embryos. Development 110: 1031-1040.

Reuter, R., Grunewald, B., and Leptin, M. 1993. A role for the mesoderm in endodermal migration and morphogenesis in Drosophila. Development 119: 1135-1145.

Riechmann, V., Irion, U., Wilson, R., Grosskortenhaus, A., and Leptin, M. 1997. Control of cell fates and segmentation in the Drosophila mesoderm. Development 124: 2915-2922.

Sandborn, E., Duclos, S., Messier, P., and Roberge, J. 1967. Atypical intestinal striated muscle in Drosophila melanogaster. J. Ultrastruct. Res. 18: 695-702.

Staehling-Hampton, K., Hoffmann, F.M., Baylies, M.K., Rushton, E., and Bate, M. 1994. $d p p$ induces mesodermal gene expression in Drosophila. Nature 372: 783-786.

Sun, B., Hursh, D., Jackson, D., and Beachy, P. 1995. Ultrabithorax protein is necessary but not sufficient for full activation of decapentaplegic expression in the visceral mesoderm. EMBO J. 14: 520-535.

Taylor, M. 2000. A novel Drosophila, mef2-regulated muscle gene isolated in a subtractive hybridisation-based molecular screen using small amounts of zygotic mutant RNA. Dev. Biol. 220: 37-52.

Thüringer, F., Cohen, S., and Bienz, M. 1993. Dissection of an indirect autoregulatory response of a homeotic Drosophila gene. $E M B O$ J. 12: 2419-2430.

Tonegawa, A., Funayama, N., Ueno, N., and Takahashi, Y. 1997. Mesodermal subdivision along the mediolateral axis in chicken controlled by different concentrations of BMP-4. Development 124: 1975-1984.
Tribioli, C., Frasch, M., and Lufkin, T. 1997. Bapx1: An evolutionary conserved homologue of the Drosophila bagpipe homeobox gene is expressed in splanchnic mesoderm and the embryonic skeleton. Mech. Dev. 65: 145-162.

Xu, X., Yin, Z., Hudson, J., Ferguson, E., and Frasch, M. 1998. Smad proteins act in combination with synergistic and antagonistic regulators to target Dpp responses to the Drosophila mesoderm. Genes \& Dev. 12: 2354-2370.

Yang, X., van Beest, M., Clevers, H., Jones, T., Hursh, D., and Mortin, M. 2000. decapentaplegic is a direct target of dTcf repression in the Drosophila visceral mesoderm. Development 127: 3695-3702.

Yin, Z. and Frasch, M. 1998. Regulation and function of tinman during dorsal mesoderm induction and heart specification in Drosophila. Dev. Genet. 22: 187-200.

Yu, X., Hoppler, S., Eresh, S., and Bienz, M. 1996. decapentaplegic, a target gene of the wingless signalling pathway in the Drosophila midgut. Development 122: 849-858.

Zaffran, S. and Frasch, M. 2000. Barbu: An $E(s p l) m 4 / m(\alpha)$-related gene that antagonizes Notch signaling and is required for the establishment of ommatidial polarity. Development 127: 1115-1130. 


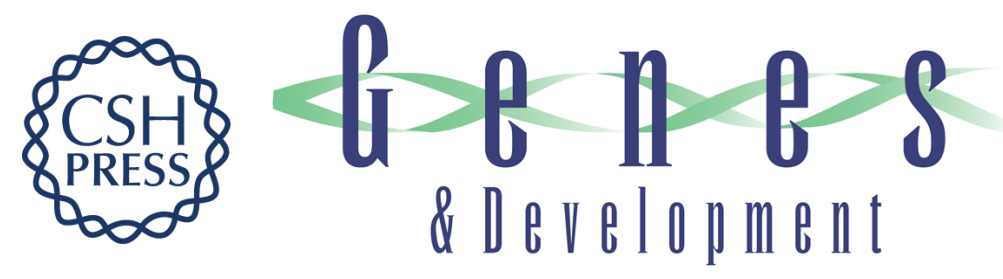

\section{biniou (FoxF), a central component in a regulatory network controlling visceral mesoderm development and midgut morphogenesis in Drosophila}

Stephane Zaffran, Axel Küchler, Hsiu-Hsiang Lee, et al.

Genes Dev. 2001, 15:

Access the most recent version at doi:10.1101/gad.917101

References This article cites 47 articles, 22 of which can be accessed free at:

http://genesdev.cshlp.org/content/15/21/2900.full.html\#ref-list-1

License

Email Alerting Receive free email alerts when new articles cite this article - sign up in the box at the top

Service right corner of the article or click here.

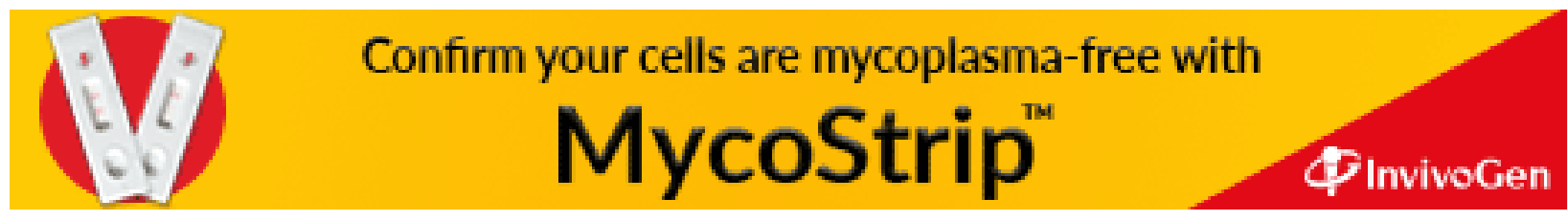

